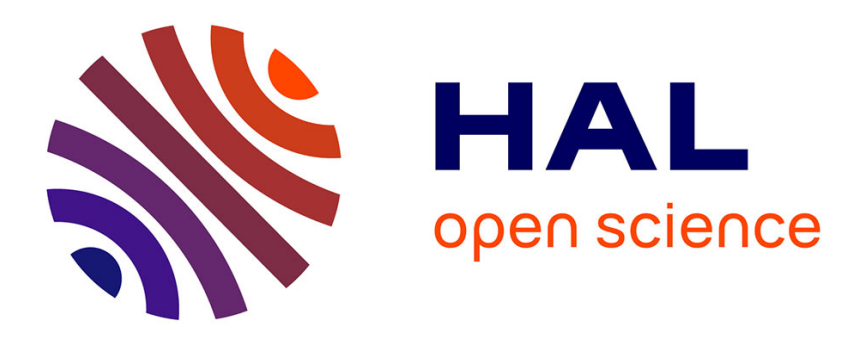

\title{
Control of Stackelberg for coupled parabolic equations
}

\author{
Moumini Kéré, Michelle Mercan, Gisèle Mophou
}

\section{To cite this version:}

Moumini Kéré, Michelle Mercan, Gisèle Mophou. Control of Stackelberg for coupled parabolic equations. Journal of Dynamical and Control Systems, 2017, 23, pp.709-733. 10.1007/s10883-016-9354-3 . hal-02548551

\section{HAL Id: hal-02548551 \\ https://hal.science/hal-02548551}

Submitted on 20 Apr 2020

HAL is a multi-disciplinary open access archive for the deposit and dissemination of scientific research documents, whether they are published or not. The documents may come from teaching and research institutions in France or abroad, or from public or private research centers.
L'archive ouverte pluridisciplinaire HAL, est destinée au dépôt et à la diffusion de documents scientifiques de niveau recherche, publiés ou non, émanant des établissements d'enseignement et de recherche français ou étrangers, des laboratoires publics ou privés. 


\title{
Control of Stackelberg for coupled parabolic equations
}

\author{
Moumini KÉRÉ * Michelle MERCAN ${ }^{\dagger}$ Gisèle MOPHOU ${ }^{\ddagger}$
}

February 1, 2018

\begin{abstract}
We consider the Stackelberg problem for coupled parabolic equations with a finite number of constraints on one of the states. This notion assumes that we have two controls to determine. The first control is supposed to bring the solution of the coupled system subjected to a finite number of constraints at rest at time zero while the second expresses that the states do not move too far from given states. The results are achieved by means of an observability inequality of Carleman adapted to the constraints.
\end{abstract}

Key-words : Null-controllability, Coupled system, Carleman inequalities, observability inequality.

AMS Subject Classification 35K05, 35K15, 35K20, 49J20, 93B05

\section{Introduction}

Let $N \in \mathbb{N}^{*}$ and $\Omega$ be a bounded open subset of $\mathbb{R}^{N}$ with boundary $\Gamma$ of class $\mathcal{C}^{2}$. For $T>0$, we set $Q=\Omega \times(0, T), \Sigma=\Gamma \times(0, T)$ and $\omega_{T}=\omega \times(0, T)$ where $\omega$ is a sub-domain compactly embedded in $\Omega$. We consider the following system:

*Département de Sciences Exactes, Institut Des Sciences, Ouagadougou, Burkina Faso 01 BP 1757 ouaga 01- Laboratoire MAINEGE, Université Ouaga 3S, O6 BP 10347 Ouagadougou 06, Burkina Faso, email : moumik3000@gmail.com

†Université des Antilles, Campus Fouillole, 97159 Pointe-à-Pitre Guadeloupe (FWI), email : michelle.mercan@univ-ag.fr

$\ddagger$ Université des Antilles, Campus Fouillole, 97159 Pointe-à-Pitre Guadeloupe (FWI)- Laboratoire MAINEGE, Université Ouaga 3S, 06 BP 10347 Ouagadougou 06, Burkina Faso, email : Gisele.Mophou@univ-ag.fr 


$$
\left\{\begin{aligned}
\frac{\partial y_{1}}{\partial t}-\Delta y_{1}+a y_{1}+b y_{2} & =l+k \chi_{\omega} & & \text { in } Q, \\
\frac{\partial y_{2}}{\partial t}-\Delta y_{2}+c y_{2}+d y_{1} & =0 & & \text { in } Q, \\
y_{1}=y_{2} & =0 & & \text { on } \Sigma, \\
y_{1}(0)=y_{2}(0) & =0 & & \text { in } \Omega,
\end{aligned}\right.
$$

where the controls $l$ and $k$ belong to $L^{2}(Q), \chi_{\omega}$ denotes the characteristic function of the set $\omega$ and

$$
a, b, c, d \in L^{\infty}(Q), \text { with } d \geq \nu>0 \text { in } \omega_{T} .
$$

Under the assumptions on the data, we know that system (1) has an unique solution $\left(y_{1}, y_{2}\right)=\left(y_{1}(l, k), y_{2}(l, k)\right) \in\left(C\left([0, T] ; L^{2}(\Omega)\right) \cap L^{2}\left((0, T) ; H_{0}^{1}(\Omega)\right)\right)^{2}($ See. [20]). Moreover, if we set $\|a, b, c, d\|_{L^{\infty}(Q)}^{2}=\|a\|_{L^{\infty}(Q)}^{2}+\|b\|_{L^{\infty}(Q)}^{2}+\|c\|_{L^{\infty}(Q)}^{2}+$ $\|d\|_{L^{\infty}(Q)}^{2}$, there exists a positive constant $C=C\left(\Omega, T,\|a, b, c, d\|_{L^{\infty}(Q)}\right)$ such that

$$
\left\|y_{1}\right\|_{L^{2}\left(0, T, H_{0}^{1}(\Omega)\right)}+\left\|y_{2}\right\|_{L^{2}\left(0, T, H_{0}^{1}(\Omega)\right)} \leq C\left(\|l\|_{L^{2}(Q)}+\|k\|_{L^{2}\left(\omega_{T}\right)}\right) .
$$

The Stackelberg leadership model is a strategic game in economics in which two firms compete on the market of the same product. The first to act must integrate the reaction of the other company in the choices it makes in the amount of product that it decides to put on the market. Following this notion, we want to control system (1) by acting with two controls. To this end, we set

$$
H_{\theta}=\left\{h \in L^{2}(Q) \text { such that } \theta h \in L^{2}(Q)\right\},
$$

where $\theta$ is positive function precisely defined later on by (14). Then, it easy to prove that $H_{\theta}$ endowed with the norm

$$
\|h\|_{H_{\theta}}^{2}=(\theta h, \theta h)_{L^{2}(Q)}=\int_{Q}|\theta h|^{2} d x d t, \quad \forall h \in H_{\theta}
$$

is a Hilbert space. We consider the following problems:

Problem 1.1 Given $a, c, b, d \in L^{\infty}(Q)$ with $d \geq \nu>0$ in $\omega_{T}$ and $e_{i} \in L^{2}(Q)$, $1 \leq i \leq M$ such that

$$
\left(e_{i} \chi_{\omega}\right)_{1 \leq i \leq M} \text { are linearly independent. }
$$

For any $l \in H_{\theta}$, find a control $k=k(l) \in L^{2}\left(\omega_{T}\right)$ such that $\left(y_{1}, y_{2}\right)$ solution of (1) satisfies

$$
y_{1}(T)=y_{2}(T)=0 \text { in } \Omega
$$

and

$$
\int_{Q} y_{2} e_{i} d x d t=0,1 \leq i \leq M
$$


Problem 1.2 Assume that the control $k=k(l)$, solution of Problem 1.1 exists. Let $y=\left(y_{1}(l, k(l)), y_{2}(l, k(l))\right)$ be the solution of (1) corresponding to $k=k(l)$. Let also $\mathcal{U}_{a d}$ be a nonempty closed convex subset of $H_{\theta}$ and $\left(z_{d}^{1}, z_{d}^{2}\right) \in\left(L^{2}(Q)\right)^{2}$. Find $\hat{l} \in \mathcal{U}_{a d}$ such that

$$
J(\hat{l})=\inf _{l \in \mathcal{U}_{a d}} J(l)
$$

where

$$
J(l)=\frac{1}{2}\left\|y_{1}(l, k(l))-z_{d}^{1}\right\|_{L^{2}(Q)}^{2}+\frac{1}{2}\left\|y_{2}(l, k(l))-z_{d}^{2}\right\|_{L^{2}(Q)}^{2}+\frac{N}{2}\|l\|_{H_{\theta}}^{2} .
$$

If the controls $\mathrm{l}$ and $\mathrm{k}$ exist, then the linear system (1) is controllable in the sense of Stackelberg. Following this strategy, the control $l$ is the Leader and $k$ is the Follower.

Problem 1.2 is a classical optimal control problem [3] whereas Problem 1.1 is a simultaneous controllability problem with state constraints.

There are some works on simultaneous controllability. In [17], D.L. Russell studied a simultaneous controllability for two wave equations; one with boundary control of Dirichlet type, the other of Neumann type in order to controlling the time evolution of electromagnetic fields independent of the axial coordinate. Using the Hilbert Uniqueness Method (HUM) developed by J. L. Lions in [5], G.O. Antunes et al. in [18] proved under suitable condition on final time that the boundary simultaneous controllability for a system of equations that constitutes a model of dynamical elasticity for incompressible materials holds. Following the same method, B.V. Kapitonov and G.P. Menzala in [19] proved the exact null simultaneous controllability for Maxwell equations system and for a wave equation vector with a pressure term. In [21], F. Ammar Khodja et al. studied the local simultaneous null controllability of a coupled system of semi-linear heat equations. They showed the existence of simultaneous control for a linear coupled system which is derived from the initial system. Then, by a argument of the fixed point, they established the existence of the solution of local simultaneous null controllability problems for the semi linear coupled system.

Recently, C. Louis-Rose [15] studied the simultaneous controllability problem with constraint on the control of a coupled system of linear heat equations that each had the same control. She initially turned the system into an equivalent system with the control acting only in a single equation, using a suitable change of variable. Then she solved the problem of simultaneous controllability of this new system using a Carleman inequality adapted to the constraint. This result was generalized to simultaneous null controllability for a coupled system of reaction-diffusion with a finite number of state constraints by Peng Gao [22].

The notion of controllability in the sense of Stackelberg was initiated by O. Nakoulima [8]. Actually, the author was interested in the concept of hierarchical control for a backward heat equation. This concept assumes to have two controls to determine: one of null controllability type with constraint on the control, called Follower, and the other of optimal control type, called Leader. The results 
were achieved by means of a Carleman inequality adapted to the constraint and were applied to a problem of discriminating sentinels (see [4] for the notion of sentinel). In $[9,10]$, M. Mercan revisited the notion of controllability in the sense of Stackelberg given by O. Nakoulima [8] by choosing the Follower of minimal norm. This new notion is then applied by M. Mercan and O. Nakoulima in [13] on the controllability of a two-stroke problem with constraint on the states. The results were obtained by means of Carleman inequality adapted to the constraints. In this paper, motivated by all the above works, we investigate the controllability in the sense of Stackelberg to a system of coupled parabolic equations with constraints on one state. The main results is as follows:

Theorem 1.3 Let $\Omega$ be a bounded open subset of $\mathbb{R}^{N}$ with boundary $\Gamma$ of class $\mathcal{C}^{2}$ and $\omega$ be a sub-domain compactly embedded in $\Omega$. Let $a, c, b, d \in L^{\infty}(Q)$ with $d \geq \nu>0$ in $\omega_{T}$ and $e_{i} \in L^{2}(Q), 1 \leq i \leq M$ be such that (6) holds. Let also $H_{\theta}$ be the Hilbert space defined by (4) and (5). Then there exists a positive real function $\theta$ (a precise definition of $\theta$ will be given later on) such that for any function $l \in H_{\theta}$, there exists a unique control $k=k(l) \in L^{2}(Q)$ such that $\left(k(l), y=\left(y_{1}(l, k(l)), y_{2}(l, k(l))\right)\right)$ is the solution of the null controllability problem with constraints on the state (1), (7), (8). Moreover,

$$
k=P \rho_{1} \chi_{\omega}-\rho_{1} \chi_{\omega}-u_{0} \chi_{\omega},
$$

where $P$ is the orthogonal projection operator from $L^{2}\left(\omega_{T}\right)$ into $K$ ( a precise definition of $K$ will be given later on $), u_{0}=u_{0}(l) \in \frac{1}{\theta} K$ and $\left(\rho_{1}, \rho_{2}\right)=$ $\left(\rho_{1}(l), \rho_{2}(l)\right)$ satisfies:

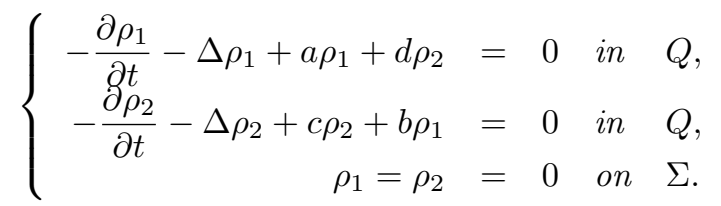

Theorem 1.4 Assume that the hypotheses of Theorem 1.3 holds. Then there exists $\left(p_{1}, p_{2}\right) \in\left(C\left([0, T] ; L^{2}(\Omega)\right) \cap L^{2}\left((0, T) ; H_{0}^{1}(\Omega)\right)\right)^{2}$ such that the minimization problem (9) admits a unique solution $\hat{l}$ which is characterized by the following optimality condition

$$
\left(\Lambda^{-1}\left(\frac{1}{\theta} p_{1}+\frac{1}{\theta} F^{*}\left(p_{1} \chi_{\omega}\right)\right)+N \hat{l}, l-\hat{l}\right)_{H_{\theta}} \geq 0, \quad \forall l \in \mathcal{U}_{a d}
$$

where $F$ is a linear and continuous application from $H_{\theta}$ to $L^{2}(Q)$ precisely defined later on by (101), $F^{*}$ is the adjoint of $F$ and $\left(p_{1}, p_{1}\right)$ is solution of

$$
\left\{\begin{array}{rlll}
-\frac{\partial p_{1}}{\partial t}-\Delta p_{1}+a p_{1}+d p_{2} & =\hat{y}_{1}-z_{d}^{1} & \text { in } \quad Q \\
-\frac{\partial p_{2}}{\partial t}-\Delta p_{2}+c p_{2}+b p_{1} & =\hat{y}_{2}-z_{d}^{2} & \text { in } \quad Q \\
p_{1}=p_{2} & =0 & & \text { on } \Sigma \\
p_{1}(T)=0 ; p_{2}(T) & =0 & \text { in } \Omega
\end{array}\right.
$$


with $\left(\hat{y}_{1}, \hat{y}_{2}\right) \in\left(C\left([0, T] ; L^{2}(\Omega)\right) \cap L^{2}\left((0, T) ; H_{0}^{1}(\Omega)\right)\right)^{2}$, the solution to (1) corresponding to the control $\hat{l}$.

The paper is organized as follows. Section 2 is devoted to the study of Problem 1.1. In this section, we prove the equivalence between the null controllability problem with constraints on the state and a null controllability with constraint on the control. Then, we establish a Carleman inequality adapted to the constraints and prove Theorem 1.3. We prove Theorem 1.4 in section 3.

\section{Study of Problem 1}

We first prove that the null controllability with constraint on the state is equivalent to a null controllability with constraint on the control. Then we solve the equivalent problem using an adapted Carleman inequality and a penalization method. This allows to prove Theorem 1.3.

\subsection{Equivalence between the null controllability with con- straint on the state and a null controllability with con- straint on the control.}

In this section, we show the equivalence between the null controllability problem with state constraints and a null controllability problem with constraint on the control. But before going further, we need some preliminary results.

So, let $\omega^{\prime} \Subset \omega$ and $\psi \in C^{2}(\bar{\Omega})$ a function such that

$$
\left\{\begin{array}{c}
\min \left\{|\nabla \psi(x)|, x \in \overline{\Omega \backslash \omega^{\prime}}\right\}>0 \\
\frac{\partial \psi}{\partial \nu} \leq 0 \text { on } \Gamma
\end{array}\right.
$$

where $\nu$ denotes the outward unit to $\Gamma$. Suppose moreover that $\psi$ satisfies

$$
\min \{\psi(x), x \in \bar{\Omega}\} \geq \max \left\{\frac{3}{4}\|\psi\|_{L^{\infty}(\Omega)}, \ln 3\right\} .
$$

Such function $\psi$ was considered by A. Fursikov and O. Imanuvilov [1]. For all $\lambda>0, \tau>0$, we set

$$
\begin{gathered}
\rho(x, t)=\frac{e^{\lambda \psi(x)}}{t(T-t)}, \quad(x, t) \in Q, \\
\phi(x, t)=\tau \frac{e^{\frac{4}{3^{\lambda\|\psi\|_{L} \infty(\Omega)}}-e^{\lambda \psi(x)}}}{t(T-t)}, \quad(x, t) \in Q
\end{gathered}
$$

and

$$
\frac{1}{\theta^{2}}(x, t)=e^{-2 \phi(x, t)}, \quad(x, t) \in Q
$$


Then $\frac{1}{\theta} \in L^{\infty}(Q)$ because $\phi(x, t)>0$ for all $(x, t) \in Q$.

Lemma 2.1 Let $\theta$ be defined by (14) and the functions $\left(e_{i}\right)_{1 \leq i \leq M} \in\left(L^{2}(Q)\right)^{M}$ be such that (6) holds. Let $a, c, b, d \in L^{\infty}(Q)$ with $d \geq \nu>0$ in $\omega_{T}$. Let also $\left(p_{i}, q_{i}\right) \in\left(C\left([0, T] ; L^{2}(\Omega)\right) \cap L^{2}\left((0, T) ; H_{0}^{1}(\Omega)\right)\right)^{2}$ be solution of:

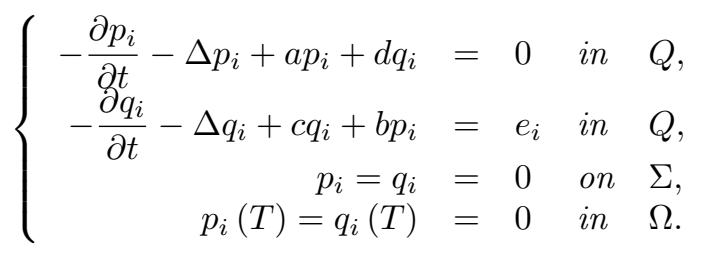

Then the families $\left(p_{1} \chi_{\omega}, p_{2} \chi_{\omega}, \ldots, p_{M} \chi_{\omega}\right)$ and $\left(\frac{1}{\theta} p_{1} \chi_{\omega}, \frac{1}{\theta} p_{2} \chi_{\omega}, \ldots, \frac{1}{\theta} p_{M} \chi_{\omega}\right)$ are linearly independent.

Proof. Let $\left(\alpha_{i}\right)_{i=1, \ldots, M} \in \mathbb{R}^{M}$ be such that $k=\sum_{i=1}^{M} \alpha_{i} p_{i}=0$ in $\omega_{T}$. Let $z=\sum_{i=1}^{M} \alpha_{i} q_{i}$ in $\omega_{T}$. From (15) we have

$$
\left\{\begin{array}{rlrl}
-\frac{\partial k}{\partial t}-\Delta k+a k+d z & =0 & \text { in } Q \\
-\frac{\partial z}{\partial t}-\Delta z+c z+b k & =\sum_{i=1}^{M} \alpha_{i} e_{i} & & \text { in } Q \\
k=z & =0 & & \text { on } \Sigma, \\
k(T)=z(T) & =0 & & \text { in } \Omega .
\end{array}\right.
$$

As $k=0$ in $\omega_{T}$ and $d \neq 0$ in $\omega_{T}$, equality $(16)_{1}$ give us $z=0$ in $\omega_{T}$. Then, using the fact that $k=z=0$ in $\omega_{T}$ in $(16)_{2}$, we obtain $0=\sum_{i=1}^{M} \alpha_{i} e_{i}$ in $\omega_{T}$. Consequently, assumption (6) allows us to conclude that $\alpha_{i}=0$ for all $1 \leq$ $i \leq M$. Analogously, we prove that the family $\left(\frac{1}{\theta} p_{i} \chi_{\omega}\right)_{i=1, \ldots, M}$ is linearly independent.

We set

$$
\begin{aligned}
K & =\operatorname{Span}\left(p_{1} \chi_{\omega}, p_{2} \chi_{\omega}, \cdots, p_{M} \chi_{\omega}\right) \\
K_{\theta} & =\frac{1}{\theta} K .
\end{aligned}
$$

Lemma 2.2 Let $\theta$ be defined by (14) and $\left(e_{i}\right)_{1 \leq i \leq M} \in\left(L^{2}(Q)\right)^{M}$ such that (6) holds. Let also $\left(p_{i}, q_{i}\right)$ be defined by (15). Set

$$
A_{\theta}=\left(\int_{Q} \frac{1}{\theta} p_{i} p_{j} \chi_{\omega}\right)_{1 \leq i, j \leq M}
$$


Then, there exists $\delta>0$ such that

$$
\left(A_{\theta} X, X\right)_{\mathbb{R}^{M}} \geq \delta\|X\|_{\mathbb{R}^{M}}^{2},
$$

where

$$
\left(A_{\theta} X, X\right)_{\mathbb{R}^{M}}=\int_{\omega_{T}} \frac{1}{\theta}\left(\sum_{i=1}^{M} X_{i} p_{i}\right)\left(\sum_{i=1}^{M} X_{j} p_{j}\right) d x d t
$$

and

$$
X=\left(X_{1}, \ldots, X_{M}\right) \in \mathbb{R}^{M} .
$$

Proof. One proceeds as in [22].

Proposition 2.1 Let $\theta$ be defined by (14) and $H_{\theta}$ be defined by (4) and (5). Let also $K$ and $K_{\theta}$ be defined by (17) and (18) respectively. Then the null controllability problem with constraints on the state (1), (7), (8) is equivalent to the following null controllability problem with constraint on control: Given $a, c, b, d \in L^{\infty}(Q)$ with $d \geq \nu>0$ in $\omega_{T}, l \in H_{\theta}$ and $u_{0}=u_{0}(l) \in K_{\theta}$, find

$$
v=v(l) \in K^{\perp}
$$

such that $\left(y_{1}, y_{2}\right)$, solution of

$$
\left\{\begin{aligned}
\frac{\partial y_{1}}{\partial t}-\Delta y_{1}+a y_{1}+b y_{2} & =l+\left(v-u_{0}\right) \chi_{\omega} & & \text { in } Q, \\
\frac{\partial y_{2}}{\partial t}-\Delta y_{2}+c y_{2}+d y_{1} & =0 & & \text { in } Q, \\
y_{1}=y_{2} & =0 & & \text { on } \Sigma, \\
y_{1}(0)=y_{2}(0) & =0 & & \text { in } \Omega
\end{aligned}\right.
$$

satisfies

$$
y_{1}(T)=y_{2}(T)=0 \text { in } \Omega .
$$

Proof. Assume that $\left(y_{1}, y_{2}\right)$ is solution of (1), (7), (8). Multiplying (15) by $y_{1}$ and $(15)_{2}$ by $y_{2}$ and integrating by parts over $Q$, we get

$$
\begin{gathered}
\int_{Q} p_{i}\left(\frac{\partial y_{1}}{\partial t}-\Delta y_{1}+a y_{1}\right) d x d t+\int_{Q} d q_{i} y_{1} d x d t=\int_{Q} e_{i} y_{2} d x d t \\
\int_{Q} q_{i}\left(\frac{\partial y_{2}}{\partial t}-\Delta y_{2}+c y_{2}\right) d x d t+\int_{Q} b p_{i} y_{2} d x d t=0 .
\end{gathered}
$$

Adding the resulting identities and using (8), we obtain that

$$
-\int_{Q} l p_{i} d x d t=\int_{\omega_{T}} k p_{i} d x d t .
$$

Since the matrix $A_{\theta}$ defined by (19) is symmetric positive definite, there exists a unique $u_{0}=u_{0}(l) \in K_{\theta}$ such that

$$
\int_{Q} l p_{i} d x d t=\int_{\omega_{T}} u_{0} p_{i} d x d t
$$


Combining (24) and (25) we deduce that

$$
\int_{\omega_{T}} u_{0} p_{i} d x d t=-\int_{\omega_{T}} k p_{i} d x d t
$$

Therefore

$$
\int_{\omega_{T}}\left(u_{0}+k\right) p_{i} d x d t=0, \quad 1 \leq i \leq M .
$$

This means that $u_{0}+k=v \in K^{\perp}$. It then follows that $k=v-u_{0}$. Hence, replacing $k$ by $v-u_{0}$ in (1) we obtain (22) .

Conversely, assume that $\left(v,\left(y_{1}, y_{2}\right)\right)$ is solution of $(21),(22)$ and (23). Multiplying $(15)_{1}$ by $y_{1}$ and $(15)_{2}$ by $y_{2}$ and integrate by parts over $Q$, we get

$$
\begin{gathered}
\int_{Q} p_{i}\left(\frac{\partial y_{1}}{\partial t}-\Delta y_{1}+a y_{1}\right) d x d t+\int_{Q} d q_{i} y_{1} d x d t=0 \\
\int_{Q} q_{i}\left(\frac{\partial y_{2}}{\partial t}-\Delta y_{2}+c y_{2}\right) d x d t+\int_{Q} b p_{i} y_{2} d x d t=\int_{Q} e_{i} y_{2} d x d t
\end{gathered}
$$

Adding the resulting identities, we obtain

$$
\int_{Q} p_{i}\left(l+\left(v-u_{0}\right) \chi_{\omega}\right) d x d t=\int_{Q} y_{2} e_{i} d x d t .
$$

Since $v \in K^{\perp}$, we have

$$
\int_{Q} p_{i}\left(l-u_{0} \chi_{\omega}\right) d x d t=\int_{Q} y_{2} e_{i} d x d t
$$

which in view of $(25)$, yields

$$
\int_{Q} y_{2} e_{i} d x d t=0
$$

We thus have proved that the pair $(k, y)$ with $k=v-u_{0}$ and $y=\left(y_{1}, y_{2}\right)$ satisfies the null controllability problem with state constraints (1), (7), (8).

From now on, we use $C(X)$ to denote a positive constant whose value varies from a line to another but depends on $\mathrm{X}$.

Proposition 2.2 Let $\theta$ be defined by (14) and $l \in H_{\theta}$. Let also $p_{i}$ and $u_{0}$ be respectively defined by (15) and (25). Then there exists a positive constant $C=C\left(\Omega,\|(a, b, c, d)\|_{\infty}, \sum_{i=1}^{M}\left\|e_{i}\right\|_{L^{2}(Q)}^{2}\right)$ such that

$$
\begin{aligned}
\left\|\theta u_{0}\right\|_{L^{2}\left(\omega_{T}\right)} & \leq C\|l\|_{H_{\theta}}, \\
\left\|u_{0}\right\|_{L^{2}\left(\omega_{T}\right)} & \leq C\|l\|_{H_{\theta}} .
\end{aligned}
$$


Proof. From (25), we have

$$
\int_{\omega_{T}} u_{0} p_{i} d x d t=\int_{Q} l p_{i} d x d t, 1 \leq i \leq M
$$

Since $u_{0}(l) \in \operatorname{Span}\left\{\frac{1}{\theta} p_{1} \chi_{\omega}, \ldots, \frac{1}{\theta} p_{M} \chi_{\omega}\right\}$, there exists $\alpha=\left(\alpha_{1}, \ldots, \alpha_{M}\right) \in$ $\mathbb{R}^{M}$ such that

$$
u_{0}=\sum_{j=1}^{M} \alpha_{j} \frac{1}{\theta} p_{j} \chi_{\omega}
$$

Thus, replacing $u_{0}$ by $\sum_{j=1}^{M} \alpha_{j} \frac{1}{\theta} p_{j} \chi_{\omega}$ in (29), we obtain:

$$
\int_{\omega_{T}} \sum_{j=1}^{M} \alpha_{j} \frac{1}{\theta} p_{j} p_{i} d x d t=\int_{Q} l p_{i} d x d t, 1 \leq i \leq M .
$$

Consequently,

$$
\int_{\omega_{T}}\left(\sum_{j=1}^{M} \alpha_{j} \frac{1}{\theta} p_{j}\right)\left(\sum_{i=1}^{M} \alpha_{i} p_{i}\right) d x d t=\int_{Q} \theta l\left(\sum_{i=1}^{M} \alpha_{i} \frac{1}{\theta} p_{i}\right) d x d t
$$

which in view of the definition of the matrix $A_{\theta}$ given by (19) can be rewritten as

$$
A_{\theta}(X, X)=\int_{Q} \theta l\left(\sum_{i=1}^{M} \alpha_{i} \frac{1}{\theta} p_{i}\right) d x d t,
$$

with $X=\left(\alpha_{i}\right)_{1 \leq i \leq M}$. It then follows from Lemma 2.2 and the Cauchy-Schwartz inequality that

$$
\delta\|\alpha\|^{2} \leq\|l\|_{H_{\theta}} \sum_{i=1}^{M}\left|\alpha_{i}\right|\left\|\frac{1}{\theta} p_{i}\right\|_{L^{2}(Q)}
$$

Since $\frac{1}{\theta}$ is bounded, we obtain that

$$
\delta\|\alpha\|^{2} \leq C(\theta)\|l\|_{H_{\theta}} \sum_{i=1}^{M}\left|\alpha_{i}\right|\left\|p_{i}\right\|_{L^{2}(Q)} .
$$

Because $p_{i}$ is solution of (15), we have that for $1 \leq i \leq M$,

$$
\left\|p_{i}\right\|_{L^{2}(Q)} \leq C\left(\Omega,\|a, b, c, d\|_{\infty}, T\right)\left\|e_{i}\right\|_{L^{2}(Q)},
$$

which combined with (31) and the fact that $\delta>0$ gives

$$
\|\alpha\|_{\mathbb{R}^{M}} \leq \delta^{-1} C\left(\Omega, \theta,\|a, b, c, d\|_{\infty}, T\right)\|l\|_{H_{\theta}} \sqrt{\sum_{i=1}^{M}\left\|e_{i}\right\|_{L^{2}(Q)}^{2}} .
$$


Finally, from (30), we have

$$
\begin{aligned}
\left\|\theta u_{0}\right\|_{L^{2}\left(\omega_{T}\right)} & \leq \sum_{j=1}^{M}\left|\alpha_{j}\right|\left\|p_{j}\right\|_{L^{2}\left(\omega_{T}\right)} \\
& \leq C\left(\Omega, \theta,\|a, b, c, d\|_{\infty}, T\right) \sum_{j=1}^{M}\left|\alpha_{j}\right|\left\|e_{j}\right\|_{L^{2}(Q)} \\
& \leq C\left(\Omega, \theta,\|a, b, c, d\|_{\infty}, T\right)\|\alpha\|_{\mathbb{R}^{M}}\left(\sum_{i=1}^{M}\left\|e_{i}\right\|_{L^{2}(Q)}^{2}\right)^{\frac{1}{2}},
\end{aligned}
$$

on the one hand, and on the other hand,

$$
\left\|u_{0}\right\|_{L^{2}\left(\omega_{T}\right)} \leq C\left(\Omega, \theta,\|a, b, c, d\|_{\infty}, T\right)\left\|\frac{1}{\theta}\right\|_{\infty}\|\alpha\|_{\mathbb{R}^{M}}\left(\sum_{i=1}^{M}\left\|e_{i}\right\|_{L^{2}(Q)}^{2}\right)^{\frac{1}{2}} .
$$

Hence, using (32) and the fact that $\frac{1}{\theta}$ is bounded in $L^{\infty}(Q)$, and setting

$C=C\left(\Omega, \theta,\|a, b, c, d\|_{\infty}, T, \sum_{i=1}^{M}\left\|e_{i}\right\|_{L^{2}(Q)}\right)=\delta^{-1} C\left(\Omega, \theta,\|a, b, c, d\|_{\infty}, T\right)^{2} \sum_{i=1}^{M}\left\|e_{i}\right\|_{L^{2}(Q)}^{2}$,

we deduce (27) and (28).

Lemma 2.3 Assume that (2) and (6) hold. If $\left(z_{1}, z_{2}\right)$ satisfies

$$
\left\{\begin{array}{l}
-\frac{\partial z_{1}}{\partial t}-\Delta z_{1}+a z_{1}+d z_{2}=0 \quad \text { in } \omega_{T} \\
-\frac{\partial z_{2}}{\partial t}-\Delta z_{2}+c z_{2}+b z_{1}=0 \quad \text { in } \omega_{T} \\
z_{\left.1\right|_{\omega}} \in K .
\end{array}\right.
$$

Then $z_{1}$ and $z_{2}$ are identically zero in $\omega_{T}$.

Proof. Since $z_{\left.1\right|_{\omega}} \in K$, there exists $\left(\alpha_{i}\right)_{1 \leq i \leq M} \in \mathbb{R}^{M}$ such that $z_{1 \mid \omega}=$ $\sum_{i=1}^{M} \alpha_{i} p_{i} \chi_{\omega}$. In view of $(15)_{1}$, this gives

$$
-\frac{\partial z_{1}}{\partial t}-\Delta z_{1}+a z_{1}+d \sum_{i=1}^{M} \alpha_{i} q_{i}=0 \text { in } \omega_{T}
$$

Since $d \neq 0$ in $\omega_{T}$, we deduce from $(33)_{1}$ and (34) that

$$
z_{2}=\sum_{i=1}^{M} \alpha_{i} q_{i} \text { in } \omega_{T}
$$


Then, it follows from $(15)_{2}$ and $(33)_{2}$ that

$$
\sum_{i=1}^{M} \alpha_{i} e_{i}=-\frac{\partial z_{2}}{\partial t}-\Delta z_{2}+c z_{2}+b z_{1}=0 \text { in } \omega_{T}
$$

Assumption (6) allows us to conclude that $\alpha_{i}=0,1 \leq i \leq M$. Hence, $z_{1}=z_{2}=0$ in $\omega_{T}$.

To solve Problem (21) - (23), we need a Carleman inequality adapted to the constraint on the control.

\subsection{Adapted Carleman inequality}

In this section we use the global Carleman due to A. Fursikov in [2] (Theorem 7.1 p. 288) to establish an adapted Carleman inequality necessary for resolution of the null controllability with constraint on the control (21)-(23).

Theorem 2.1 Let $\psi, \rho$ and $\phi$ be defined by (11), (12) and (13) respectively. Then there exist $\lambda_{0}=\lambda_{0}\left(\Omega, \omega^{\prime}\right)>0, \tau_{0}=\tau_{0}\left(\Omega, \omega^{\prime}, T\right)>0$, and a positive constant $C_{0}=C_{0}\left(\Omega, \omega^{\prime}\right)$ such that, for any $\lambda \geq \lambda_{0}, \tau \geq \tau_{0}$ and $s \geq-3$, the following inequality

$$
\begin{aligned}
& \int_{Q}\left(\frac{1}{\lambda}\left|\frac{\partial z}{\partial t}\right|^{2}+\frac{1}{\lambda}|\Delta z|^{2}+\lambda \tau^{2} \rho^{2}|\nabla z|^{2}+\lambda^{4} \tau^{4} \rho^{4}|z|^{2}\right) \rho^{2 s-1} e^{-2 \phi} d x d t \\
& \leq C_{0}\left(\tau \int_{Q}\left|\frac{\partial z}{\partial t} \pm \Delta z\right|^{2} \rho^{2 s} e^{-2 \phi} d x d t+\lambda^{4} \tau^{4} \int_{0}^{T} \int_{\omega^{\prime}}|z|^{2} \rho^{2 s+3} e^{-2 \phi} d x d t\right)
\end{aligned}
$$

holds for any $z$ satisfying the Dirichlet homogeneous condition and such that the right-hand side of the identity (35) is finite.

We adopt the following notations:

$$
\left\{\begin{aligned}
\mathcal{W}=\left\{\left(\varphi_{1}, \varphi_{2}\right) \in C^{\infty}(\bar{Q}) \times C^{\infty}(\bar{Q}), \varphi_{1}=\varphi_{2}=0 \text { on } \Sigma\right\} & \\
L_{0} \varphi & =\frac{\partial \varphi}{\partial t}-\Delta \varphi \\
L_{0}^{*} \varphi & =-\frac{\partial \varphi}{\partial t}-\Delta \varphi \\
\mathcal{M}(\varphi, \sigma) & =L_{0}^{*} \varphi+a \varphi+d \sigma \\
\mathcal{N}(\varphi, \sigma) & =L_{0}^{*} \sigma+c \sigma+b \varphi .
\end{aligned}\right.
$$

Proposition 2.3 Let $C_{0}$ be the constant given by theorem 2.1. Assume that (2) holds, and that $\tau_{1}=\frac{T^{2}}{4}\left(\frac{4 C_{0}}{\lambda_{0}^{4}}\right)^{1 / 3}\|(a, b, c, d)\|_{\infty}^{2 / 3} \geq 1$. Then, for any $\lambda \geq \lambda_{0}, \tau \geq \tau_{1} s \geq-3$ and $r \in[0,2[$, there exists a positive constant $C=$ 


$$
\begin{aligned}
& C\left(T,\|(a, b, c, d)\|_{\infty}, r, \nu\right) \text { such that for any } \varphi=\left(\varphi_{1}, \varphi_{2}\right) \in \mathcal{W} \\
& \qquad \begin{aligned}
\int_{Q} \frac{1}{\theta^{2}}\left(\left|\varphi_{1}\right|^{2}+\left|\varphi_{2}\right|^{2}\right) d x d t \leq & C\left(\int_{\omega_{T}}\left|\varphi_{1}\right|^{2} e^{-r \phi} d x d t\right. \\
& \left.+\int_{Q} \frac{1}{\theta^{2}}\left(|\mathcal{M}(\varphi)|^{2}+|\mathcal{N}(\varphi)|^{2}\right) d x d t\right) .
\end{aligned}
\end{aligned}
$$

Proof. The proof is deduced from Lemma 2.3 and Theorem 2.4 in [15].

As $\frac{1}{\theta^{2}}$ and $e^{-r \phi}$ are bounded in $L^{\infty}(Q)$, we get this next observability inequality for all $\varphi=\left(\varphi_{1}, \varphi_{2}\right) \in \mathcal{W}$ :

$$
\begin{aligned}
\int_{Q} \frac{1}{\theta^{2}}\left(\left|\varphi_{1}\right|^{2}+\left|\varphi_{2}\right|^{2}\right) d x d t & \leq C \int_{\omega_{T}}\left|\varphi_{1}\right|^{2} d x d t \\
& +C \int_{Q}\left(|\mathcal{M}(\varphi)|^{2}+|\mathcal{N}(\varphi)|^{2}\right) d x d t
\end{aligned}
$$

where $C=C\left(T,\|(a, b, c, d)\|_{\infty}, r, \nu\right)>0$.

Remark 1 The latter inequality differs from that of C. Louis-Rose [15] because we use the fact that $e^{-r \phi}$ is bounded. This let us obtain the following adapted Carleman inequality without defining $e^{(2-r) \phi}$ as in Proposition 2.6 in [15].

Proposition 2.4 Assume that (2) and (6) hold. Assume also that the hypotheses of Proposition 2.3 hold. Then there exists a positive constant $C$ such that for any $\varphi=\left(\varphi_{1}, \varphi_{2}\right) \in \mathcal{W}$,

$$
\begin{aligned}
\int_{Q} \frac{1}{\theta^{2}}\left(\left|\varphi_{1}\right|^{2}+\left|\varphi_{2}\right|^{2}\right) d x d t \leq & C\left(\int_{\omega_{T}}\left|\left(\varphi_{1}-P \varphi_{1}\right) \chi_{\omega}\right|^{2} d x d t\right. \\
& \left.+\int_{Q}\left(|\mathcal{M}(\varphi)|^{2}+|\mathcal{N}(\varphi)|^{2}\right) d x d t\right)
\end{aligned}
$$

Proof. The proof uses a well-known compactness-uniqueness argument and inequality (37). Indeed, suppose that (38) does not hold. Then, there exists $\left(\varphi_{n}=\left(\varphi_{1 n}, \varphi_{2 n}\right)\right)_{n \in \mathbb{N}^{*}} \subset \mathcal{W}$ such that

$$
\begin{aligned}
\int_{Q} \frac{1}{\theta^{2}}\left(\left|\varphi_{1 n}\right|^{2}+\left|\varphi_{2 n}\right|^{2}\right) d x d t & =1, \\
\int_{\omega_{T}}\left|\left(\varphi_{1 n}-P \varphi_{1 n}\right) \chi_{\omega}\right|^{2} d x d t & \leq \frac{1}{n}, \\
\int_{Q}\left|\mathcal{M}\left(\varphi_{n}\right)\right|^{2} d x d t & \leq \frac{1}{n}, \\
\int_{Q}\left|\mathcal{N}\left(\varphi_{n}\right)\right|^{2} d x d t & \leq \frac{1}{n} .
\end{aligned}
$$


We have

$$
\begin{aligned}
\int_{\omega_{T}} \frac{1}{\theta^{2}}\left|P \varphi_{1 n} \chi_{\omega}\right|^{2} d x d t & =\int_{\omega_{T}} \frac{1}{\theta^{2}}\left|\left(P \varphi_{1 n}-\varphi_{1 n}+\varphi_{1 n}\right) \chi_{\omega}\right|^{2} d x d t \\
& \leq 2\left(\int_{\omega_{T}} \frac{1}{\theta^{2}}\left|\left(P \varphi_{1 n}-\varphi_{1 n}\right) \chi_{\omega}\right|^{2} d x d t+\int_{\omega_{T}} \frac{1}{\theta^{2}}\left|\varphi_{1 n} \chi_{\omega}\right|^{2} d x d t\right) .
\end{aligned}
$$

Since $\frac{1}{\theta^{2}}$ is bounded, it follows from (39b) and (39a) that

$$
\int_{\omega_{T}} \frac{1}{\theta^{2}}\left|P \varphi_{1 n} \chi_{\omega}\right|^{2} d x d t \leq C
$$

Because $K$ is finite dimensional, there exists a positive real $C^{\prime}$ such that

$$
\int_{\omega_{T}}\left|P \varphi_{1 n} \chi_{\omega}\right|^{2} d x d t \leq C^{\prime}
$$

As we can write,

$$
\left\|\varphi_{1 n}\right\|_{L^{2}\left(\omega_{T}\right)} \leq\left\|\varphi_{1 n}-P \varphi_{1 n}\right\|_{L^{2}\left(\omega_{T}\right)}+\left\|P \varphi_{1 n}\right\|_{L^{2}\left(\omega_{T}\right)},
$$

using (40) and (39b), we deduce that the subsequence $\left(\varphi_{1 n} \chi_{\omega}\right)_{n \in \mathbb{N}^{*}}$ is bounded in $L^{2}\left(\omega_{T}\right)$. Consequently, we can extract a subsequence of $\left(\varphi_{1 n}\right)_{n \mathbb{N}^{*}}$, still denoted $\left(\varphi_{1 n}\right)_{n \in \mathbb{N}^{*}}$, such that

$$
\varphi_{1 n} \rightarrow \varphi_{1} \text { weakly in } L^{2}\left(\omega_{T}\right) .
$$

Hence, from the continuity and the compactness of $P$, we deduce that

$$
P \varphi_{1 n} \rightarrow P \varphi_{1} \text { strongly in } L^{2}\left(\omega_{T}\right) .
$$

In view of $(39 \mathrm{~b})$, we have that

$$
\left(\varphi_{1 n}-P \varphi_{1 n}\right) \rightarrow 0 \text { strongly in } L^{2}\left(\omega_{T}\right) .
$$

It then follows from (42) and (43) that

$$
\varphi_{1 n} \rightarrow P \varphi_{1} \text { strongly in } L^{2}\left(\omega_{T}\right),
$$

which combining with (41) implies

$$
P \varphi_{1}=\varphi_{1} .
$$

This means on the one hand that

$$
\varphi_{1} \in K,
$$

and on the other hand that

$$
\varphi_{1 n} \rightarrow \varphi_{1} \text { strongly in } L^{2}\left(\omega_{T}\right)
$$


since (44) holds.

Now, in view of (39a) we have

$$
\int_{Q} \frac{1}{\theta^{2}}\left|\varphi_{i n}\right|^{2} d x d t \leq 1, i=1,2 .
$$

Using the definition of $\frac{1}{\theta^{2}}$, we deduce that $\left(\varphi_{i n}\right)_{n}, i=1,2$ is bounded in $L^{2}((\mu, T-\mu) \times \Omega), \forall \mu>0$. Therefore, there exists $\left(\varphi_{1}, \varphi_{2}\right) \in\left[L^{2}((\mu, T-\mu) \times \Omega)\right]^{2}$ such that

$$
\varphi_{i n} \rightarrow \varphi_{i} \text { weakly in } L^{2}((\mu, T-\mu) \times \Omega), i=1,2 .
$$

Consequently,

$$
\varphi_{\text {in }} \rightarrow \varphi_{i} \text { in } \mathcal{D}^{\prime}(Q), i=1,2 \text {. }
$$

This implies that

$$
\mathcal{M}\left(\varphi_{n}\right) \rightarrow M\left(\varphi_{1}, \varphi_{2}\right) \text { in } \mathcal{D}^{\prime}(Q)
$$

and

$$
\mathcal{N}\left(\varphi_{n}\right) \rightarrow N\left(\varphi_{1}, \varphi_{2}\right) \text { in } \mathcal{D}^{\prime}(Q) .
$$

From $(39 \mathrm{c})$ and $(39 \mathrm{~d})$, we have that

$$
\mathcal{M}\left(\varphi_{n}\right) \rightarrow 0 \text { strongly in } L^{2}(Q)
$$

and

$$
\mathcal{N}\left(\varphi_{n}\right) \rightarrow 0 \text { strongly in } L^{2}(Q) .
$$

It then follows from the uniqueness of the limit that

$$
\mathcal{M}\left(\varphi_{1}, \varphi_{2}\right)=\mathcal{N}\left(\varphi_{1}, \varphi_{2}\right)=0 \text { in } Q .
$$

Using (45), (49) and lemma 2.3, we deduce that $\varphi_{1}=\varphi_{2}=0$ in $\omega_{T}$. Finally, (46) allows us to conclude that

$$
\varphi_{1 n} \rightarrow 0 \text { strongly in } L^{2}\left(\omega_{T}\right) .
$$

Since $\varphi_{n}=\left(\varphi_{1 n}, \varphi_{2 n}\right) \in \mathcal{W}$, it follows from the observability inequality (37) that

$$
\begin{aligned}
\int_{Q} \frac{1}{\theta^{2}}\left(\left|\varphi_{1 n}\right|^{2}+\left|\varphi_{2 n}\right|^{2}\right) d x d t & \leq C \int_{\omega_{T}}\left|\varphi_{1 n}\right|^{2} d x d t \\
& +\int_{Q}\left(\left|\mathcal{M}\left(\varphi_{n}\right)\right|^{2}+\left|\mathcal{N}\left(\varphi_{n}\right)\right|^{2}\right) d x d t
\end{aligned}
$$

Then, (47), (48) and (50) yield that $\int_{Q} \frac{1}{\theta^{2}}\left(\left|\varphi_{1 n}\right|^{2}+\left|\varphi_{2 n}\right|^{2}\right) d x d t \rightarrow 0$ when $n \rightarrow+\infty$. The contradiction occurs thanks to condition (39a). The proof is complete. 


\subsection{Solution of the null controllability problem with con- straint on the control}

We proceed in tree steps.

Step 1. We prove there exists a solution $\left(v_{\theta}, y_{\theta}=\left(y_{1 \theta}, y_{2 \theta}\right)\right)$ to the null controllability problem with constraint on the control.

Consider the following symmetric bilinear form

$$
\begin{aligned}
\mathcal{B}(\varphi, \sigma) & =\int_{Q} \mathcal{M}(\varphi) M(\sigma) d x d t+\int_{Q} N(\varphi) \mathcal{N}(\sigma) d x d t \\
& +\int_{\omega_{T}}\left(\varphi_{1}-P \varphi_{1}\right)\left(\sigma_{1}-P \sigma_{1}\right) d x d t
\end{aligned}
$$

for any $\varphi=\left(\varphi_{1}, \varphi_{2}\right)$ and $\sigma=\left(\sigma_{1}, \sigma_{1}\right) \in \mathcal{W}$.

From $(38), \mathcal{B}$ is a scalar product on $\mathcal{W}$. Let

$$
W=\overline{\mathcal{W}}^{\mathcal{B}}
$$

be the completion of $\mathcal{W}$ under the norm

$$
\varphi \mapsto \sqrt{\mathcal{B}(\varphi, \varphi)} .
$$

Then the space $W=\overline{\mathcal{W}}^{\mathcal{B}}$ is a Hilbert space.

Let $H_{\theta}$ be a Hilbert space defined by (4) and $l \in H_{\theta}$. Let also $\theta$ and $u_{0}$ be respectively defined by (14) and (25). Then, using the estimate of $\theta u_{0}$ given by (27), the Carleman estimate (38), the definition of the norm on $W$ given by (53) and the Cauchy-Schwartz inequality, we prove that the linear form $\mathcal{L}$ defined on $W$ by

$$
\mathcal{L}:\left(\varphi_{1}, \varphi_{2}\right) \mapsto \int_{Q}\left(l-u_{0} \chi_{\omega}\right) \varphi_{1} d x d t
$$

is continuous on $W$.

Hence, the Riesz representation theorem allows to say that there exists a unique $\varphi_{\theta}=\left(\varphi_{1 \theta}, \varphi_{2 \theta}\right) \in W$ such that

$$
\mathcal{B}\left(\varphi_{\theta}, \varphi\right)=\mathcal{L}(\varphi), \forall \varphi \in W .
$$

Proposition 2.5 Assume that the hypotheses of Proposition 2.4 holds. Let $\varphi_{\theta} \in W$ be the unique solution of (55). Set

$$
\begin{aligned}
y_{1 \theta} & =\mathcal{M}\left(\varphi_{\theta}\right), \\
y_{2 \theta} & =\mathcal{N}\left(\varphi_{\theta}\right), \\
v_{\theta} & =-\left(\varphi_{1 \theta}-P \varphi_{1 \theta}\right) .
\end{aligned}
$$

Then $\left(v_{\theta}, y_{1 \theta}, y_{2 \theta}\right)$ is solution of $(21)-(23)$. 
Moreover,

$$
\begin{aligned}
\left\|\varphi_{\theta}\right\|_{W} & \leq C\|l\|_{H_{\theta}}, \\
\left\|y_{1 \theta}\right\|_{L^{2}(Q)} & \leq C\|l\|_{H_{\theta}}, \\
\left\|y_{2 \theta}\right\|_{L^{2}(Q)} & \leq C\|l\|_{H_{\theta}}, \\
\left\|v_{\theta}\right\|_{L^{2}(G)} & \leq C\|l\|_{H_{\theta}},
\end{aligned}
$$

where $C=C\left(\Omega,\|(a, b, c, d)\|_{\infty}, \sum_{i=1}^{M}\left\|e_{i}\right\|_{L^{2}(Q)}^{2}\right)>0$.

Proof. Since $\varphi_{1 \theta} \in L^{2}\left(\omega_{T}\right)$ and $v_{\theta}=-\left(\varphi_{1 \theta}-P \varphi_{1 \theta}\right)$, we have $v_{\theta} \in K^{\perp}$. In view of $(55)$,

$$
\begin{aligned}
& \int_{Q} \mathcal{M} \varphi_{\theta} \mathcal{M} \varphi d x d t+\int_{Q} \mathcal{N} \varphi_{\theta} \mathcal{N} \varphi d x d t+\int_{\omega_{T}}\left(\varphi_{1 \theta}-P \varphi_{1 \theta}\right)\left(\varphi_{1}-P \varphi_{1}\right) d x d t \\
& =\int_{Q}\left(l-u_{0} \chi_{\omega}\right) \varphi_{1} d x d t, \quad \forall \varphi=\left(\varphi_{1}, \varphi_{2}\right) \in W .
\end{aligned}
$$

Replacing in this latter identity $\mathcal{M} \varphi_{\theta}$ by $y_{1 \theta}, \mathcal{N} \varphi_{\theta}$ by $y_{2 \theta}$ and $\left(-\varphi_{1}+P \varphi_{1}\right) \chi_{\omega}$ by $v_{\theta}$, we deduce that

$$
\begin{aligned}
& \int_{Q} y_{1 \theta}\left(-\frac{\partial \varphi_{1}}{\partial t}-\Delta \varphi_{1}+a \varphi_{1}+d \varphi_{2}\right) d x d t+\int_{Q} y_{2 \theta}\left(-\frac{\partial \varphi_{2}}{\partial t}-\Delta \varphi_{2}+c \varphi_{2}+b \varphi_{1}\right) d x d t \\
& -\int_{\omega_{T}} v_{\theta} \varphi_{1} d x d t=\int_{Q}\left(l-u_{0} \chi_{\omega}\right) \varphi_{1} d x d t, \forall \varphi=\left(\varphi_{1}, \varphi_{2}\right) \in W
\end{aligned}
$$

Taking successively in (58) $\varphi_{2}=0$ and $\varphi_{1} \in \mathcal{D}(Q)$ and then $\varphi_{1}=0$ and $\varphi_{2} \in \mathcal{D}(Q)$, we successively obtain

$$
\int_{Q} y_{1 \theta}\left(-\frac{\partial \varphi_{1}}{\partial t}-\Delta \varphi_{1}+a \varphi_{1}\right) d x d t+\int_{Q} b y_{2 \theta} \varphi_{1} d x d t=\int_{Q}\left(l+\left(v_{\theta}-u_{0}\right) \chi_{\omega}\right) \varphi_{1} d x d t
$$

and

$$
\int_{Q} y_{2 \theta}\left(-\frac{\partial \varphi_{2}}{\partial t}-\Delta \varphi_{2}+c \varphi_{2}\right) d x d t+\int_{Q} d y_{1 \theta} \varphi_{2} d x d t=0, \quad \forall \varphi_{2} \in \mathcal{D}(Q)
$$

which after integration by parts over $Q$ gives,

$$
\begin{aligned}
& \int_{Q} \varphi_{1}\left(\frac{\partial y_{1 \theta}}{\partial t}-\Delta y_{1 \theta}+a y_{1 \theta}+b y_{2 \theta}\right) d x d t= \\
& \int_{Q}\left(l+\left(v_{\theta}-u_{0}\right) \chi_{\omega}\right) \varphi_{1} d x d t, \quad \forall \varphi_{1} \in \mathcal{D}(Q)
\end{aligned}
$$

and

$$
\int_{Q} \varphi_{2}\left(\frac{\partial y_{2 \theta}}{\partial t}-\Delta y_{2 \theta}+c y_{2 \theta}+d y_{1 \theta}\right) d x d t=0, \forall \varphi_{2} \in \mathcal{D}(Q) .
$$


It then follows that

$$
\frac{\partial y_{1 \theta}}{\partial t}-\Delta y_{1 \theta}+a y_{1 \theta}+b y_{2 \theta}=l+\left(v_{\theta}-u_{0}\right) \chi_{\omega} \text { in } Q
$$

and

$$
\frac{\partial y_{2 \theta}}{\partial t}-\Delta y_{2 \theta}+c y_{2 \theta}+d y_{1 \theta}=0 \text { in } Q .
$$

As $y_{1 \theta}, y_{1 \theta} \in L^{2}(Q)$, we have $\frac{\partial y_{1 \theta}}{\partial t} \in H^{-1}\left((0, T) ; L^{2}(\Omega)\right)$. Thus,

$$
\Delta y_{1 \theta}=\frac{\partial y_{1 \theta}}{\partial t}+a y_{1 \theta}+b y_{2 \theta}-l-\left(v_{\theta}-u_{0}\right) \chi_{\omega} \in H^{-1}\left((0, T) ; L^{2}(\Omega)\right)
$$

since $l+\left(v_{\theta}-u_{0}\right) \chi_{\omega} \in L^{2}(Q)$. Consequently,

$$
\left(y_{1 \theta}, y_{2 \theta}\right)_{\left.\right|_{\Sigma}} \in H^{-1}\left((0, T) ; H^{-1 / 2}(\Gamma)\right) \times H^{-1}\left((0, T) ; H^{-1 / 2}(\Gamma)\right)
$$

and

$$
\left(\frac{\partial y_{1 \theta}}{\partial \nu}, \frac{\partial y_{2 \theta}}{\partial \nu}\right)_{\left.\right|_{\Sigma}} \in H^{-1}\left((0, T) ; H^{-3 / 2}(\Gamma)\right) \times H^{-1}\left((0, T) ; H^{-3 / 2}(\Gamma)\right) \text { (see [6]). }
$$

Also, as $y_{1 \theta} \in L^{2}(Q), y_{2 \theta} \in L^{2}(Q)$, we have $\Delta y_{1 \theta} \in L^{2}\left((0, T) ; H^{-2}(\Omega)\right)$. Thus, we have

$$
\frac{\partial y_{1 \theta}}{\partial t}=\Delta y_{1 \theta}-a y_{1 \theta}-b y_{2 \theta}+l+\left(\gamma v_{\theta}-u_{0}\right) \chi_{\omega} \in L^{2}\left((0, T) ; H^{-2}(\Omega)\right) .
$$

Consequently,

$$
\left(y_{1 \theta}(0), y_{2 \theta}(0)\right) \in\left(H^{-1}(\Omega)\right)^{2} \text { and }\left(y_{1 \theta}(T), y_{2 \theta}(T)\right) \in\left(H^{-1}(\Omega)\right)^{2} .
$$

Multiplying (61) and (62) respectively by $\varphi_{1}$ and $\varphi_{2} \in \mathcal{C}^{\infty}(\bar{Q})$ such that $\left(\varphi_{1}, \varphi_{2}\right)=(0,0)$ on $\Sigma$, then, integrating by parts over $Q$, we obtain

$$
\begin{aligned}
\int_{Q}\left(l+\left(v_{\theta}-u_{0}\right) \chi_{\omega}\right) \varphi_{1} d x d t= & \int_{Q} b y_{2 \theta} \varphi_{1} d x d t \\
+ & \int_{Q} y_{1 \theta}\left(-\frac{\partial \varphi_{1}}{\partial t}-\Delta \varphi_{1}+a \varphi_{1}\right) d x d t \\
+ & \left\langle\varphi_{1}(T), y_{1 \theta}(T)\right\rangle_{H_{0}^{1}(\Omega), H^{-1}(\Omega)} \\
- & \left\langle\varphi_{1}(0), y_{1 \theta}(0)\right\rangle_{H_{0}^{1}(\Omega), H^{-1}(\Omega)} \\
+ & \left\langle y_{1 \theta}, \frac{\partial \varphi_{1}}{\partial \nu}\right\rangle_{H^{-1}\left((0, T) ; H^{-1 / 2}(\Gamma)\right), H_{0}^{1}\left((0, T) ; H^{1 / 2}(\Gamma)\right)} \\
& \forall \varphi_{1} \in \mathcal{C}^{\infty}(\bar{Q}) \text { such that }\left.\varphi_{1}\right|_{\Sigma}=0
\end{aligned}
$$


and

$$
\begin{aligned}
0= & \int_{Q} d y_{1 \theta} \varphi_{2} d x d t \\
+ & \int_{Q} y_{2 \theta}\left(-\frac{\partial \varphi_{2}}{\partial t}-\Delta \varphi_{2}+c \varphi_{2}\right) d x d t \\
+ & \left\langle\varphi_{2}(T), y_{2 \theta}(T)\right\rangle_{H_{0}^{1}(\Omega), H^{-1}(\Omega)} \\
- & \left\langle\varphi_{2}(0) y_{2 \theta}(0)\right\rangle_{H_{0}^{1}(\Omega), H^{-1}(\Omega)} \\
+ & \left\langle y_{2 \theta}, \frac{\partial \varphi_{2}}{\partial \nu}\right\rangle_{H^{-1}\left((0, T) ; H^{-1 / 2}(\Gamma)\right), H_{0}^{1}\left((0, T) ; H^{1 / 2}(\Gamma)\right)}, \\
& \forall \varphi_{2} \in \mathcal{C}^{\infty}(\bar{Q}) \text { such that }\left.\varphi_{2}\right|_{\Sigma}=0,
\end{aligned}
$$

which in view of relations (59) and (60) give

$$
\begin{aligned}
0= & \left\langle\varphi_{1}(T), y_{1 \theta}(T)\right\rangle_{H_{0}^{1}(\Omega), H^{-1}(\Omega)} \\
- & \left\langle\varphi_{1}(0), y_{1 \theta}(0)\right\rangle_{H_{0}^{1}(\Omega), H^{-1}(\Omega)} \\
+ & \left\langle y_{1 \theta}, \frac{\partial \varphi_{1}}{\partial \nu}\right\rangle_{H^{-1}\left((0, T) ; H^{-1 / 2}(\Gamma)\right), H_{0}^{1}\left((0, T) ; H^{1 / 2}(\Gamma)\right)} \\
& \forall \varphi_{1} \in \mathcal{C}^{\infty}(\bar{Q}) \text { such that }\left.\varphi_{1}\right|_{\Sigma}=0
\end{aligned}
$$

and

$$
\begin{aligned}
0= & \left\langle\varphi_{2}(T), y_{2 \theta}(T)\right\rangle_{H_{0}^{1}(\Omega), H^{-1}(\Omega)} \\
- & \left\langle\varphi_{2}(0) y_{2 \theta}(0)\right\rangle_{H_{0}^{1}(\Omega), H^{-1}(\Omega)} \\
+ & \left\langle y_{2 \theta}, \frac{\partial \varphi_{2}}{\partial \nu}\right\rangle_{H^{-1}\left((0, T) ; H^{-1 / 2}(\Gamma)\right), H_{0}^{1}\left((0, T) ; H^{1 / 2}(\Gamma)\right)} \\
& \forall \varphi_{2} \in \mathcal{C}^{\infty}(\bar{Q}) \text { such that }\left.\varphi_{2}\right|_{\Sigma}=0 .
\end{aligned}
$$

Choosing successively in (64) and (65) $\varphi_{i}$, such that $\varphi_{i}(0)=\varphi_{i}(T)=0$ in $\Omega$, then $\varphi_{i}(0)=0$ in $\Omega$, for $\mathrm{i}=1,2$, we obtain

$$
\begin{gathered}
y_{1 \theta}=0, \quad y_{2 \theta}=0 \text { on } \Sigma, \\
y_{1 \theta}(T)=0, \quad y_{2 \theta}(T)=0 \text { in } \Omega
\end{gathered}
$$

and then

$$
y_{1 \theta}(0)=0, \quad y_{2 \theta}(0)=\text { in } \Omega .
$$

It then follows from (61), (62), (66)-(68) that $\left(v_{\theta}, y_{1 \theta}, y_{2 \theta}\right)$ is solution of problem $(21)-(23)$.

Set $\varphi=\varphi_{\theta}=\left(\varphi_{1 \theta}, \varphi_{2 \theta}\right)$ in (55), then using definitions of the bilinear form $\mathcal{B}$, the linear form $\mathcal{L}$ and the norm on $W$ given respectively by (51), (54) and (53), we have

$$
\begin{aligned}
\left\|\varphi_{\theta}\right\|_{W}^{2} & =\int_{Q}\left(l-u_{0} \chi_{\omega}\right) \varphi_{1 \theta} d x d t \\
& =\int_{Q} \theta\left(l-u_{0} \chi_{\omega}\right) \frac{1}{\theta} \varphi_{1 \theta} d x d t \\
& \leq\left\|\theta\left(l-u_{0} \chi_{\omega}\right)\right\|_{L^{2}(Q)}\left\|\frac{1}{\theta} \varphi_{1 \theta}\right\|_{L^{2}(Q)}\left\|\leq\left(\|\theta l\|_{L^{2}(Q)}+\left\|\theta u_{0}\right\|_{L^{2}\left(\omega_{T}\right)}\right)\right\| \frac{1}{\theta} \varphi_{1 \theta} \|_{L^{2}(Q)},
\end{aligned}
$$


which in view of $(27)$ and the definition of the norm on $H_{\theta}$ gives

$$
\left\|\varphi_{\theta}\right\|_{W}^{2} \leq C\|l\|_{H_{\theta}}\left\|\frac{1}{\theta} \varphi_{1 \theta}\right\|_{L^{2}(Q)},
$$

where $C=C\left(\Omega,\|(a, b, c, d)\|_{\infty}, \sum_{i=1}^{M}\left\|e_{i}\right\|_{L^{2}(Q)}^{2}\right)$.

In view of (38), it comes that

$$
\left\|\frac{1}{\theta} \varphi_{1 \theta}\right\|_{L^{2}(Q)} \leq C\left\|\varphi_{\theta}\right\|_{W} .
$$

Combining (69) and (70), we deduce that

$$
\left\|\varphi_{\theta}\right\|_{W} \leq C\|l\|_{H_{\theta}} .
$$

In view of the definition of the norm on $W$ given by (53), we can write

$$
\left\|y_{1 \theta}\right\|_{L^{2}(Q)}^{2}+\left\|y_{2 \theta}\right\|_{L^{2}(Q)}^{2}+\left\|v_{\theta}\right\|_{L^{2}(Q)}^{2}=\left\|\varphi_{\theta}\right\|_{W}^{2} .
$$

Therefore, using (71), we obtain that

$$
\left\|y_{1 \theta}\right\|_{L^{2}(Q)}^{2}+\left\|y_{2 \theta}\right\|_{L^{2}(Q)}^{2}+\left\|v_{\theta}\right\|_{L^{2}(Q)}^{2} \leq C^{2}\|l\|_{H_{\theta}}^{2} .
$$

from which we deduce (57b), (57c) and (57d).

The adapted observability inequality(38) shows that the choice of the scalar product on $\mathcal{W}$ is not unique. Thus there exists infinitely many controls functions $v$ such that (21) - (23) hold.

Set

$$
\mathcal{E}=\left\{v \in K^{\perp} \text { such that }\left(v, y_{1}(v), y_{2}(v)\right) \text { satisfies }(21)-(23)\right\} .
$$

Then $\mathcal{E}$ is non empty and it is clearly convex and closed in $L^{2}\left(\omega_{T}\right)$. Therefore, there exists a unique control variable $\widehat{v}$ such that

$$
\|\hat{v}\|_{L^{2}\left(\omega_{T}\right)}^{2}=\min _{v \in \mathcal{E}}\|v\|_{L^{2}\left(\omega_{T}\right)}^{2} .
$$

Particularly, we have

$$
\|\hat{v}\|_{L^{2}\left(\omega_{T}\right)}^{2} \leq\left\|v_{\theta}\right\|_{L^{2}\left(\omega_{T}\right)}^{2},
$$

which in view of $(57 \mathrm{~d})$ implies that

$$
\|\hat{v}\|_{L^{2}\left(\omega_{T}\right)} \leq C\|l\|_{H_{\theta}} .
$$

In the sequel, we characterize the control $\hat{v}$ using the penalization method.

Step 2. We prove by means of penalization method that there exists a unique $\left(\widehat{v}, \widehat{y}_{1}, \widehat{y}_{2}\right)$ solution to problem of controllability with constraint on the control (21)-(23). 
So, for $\varepsilon>0$, let us consider the following optimal control problem. If $v \in K^{\perp}$ let $\left(y_{1}=y_{1}(v), y_{2}=y_{2}(v)\right)$ be the solution of (22). We know that $\left(y_{1}=y_{1}(v), y_{2}=y_{2}(v)\right) \in\left(C\left([0, T] ; L^{2}(\Omega)\right) \cap L^{2}\left((0, T) ; H_{0}^{1}(\Omega)\right)\right)^{2}$ and we can define the functional

$$
J_{\varepsilon}(v)=\frac{1}{2}\|v\|_{L^{2}\left(\omega_{T}\right)}^{2}+\frac{1}{2 \varepsilon}\left\|y_{1}(T)\right\|_{L^{2}(\Omega)}^{2}+\frac{1}{2 \varepsilon}\left\|y_{2}(T)\right\|_{L^{2}(\Omega)}^{2},
$$

The optimal control problem is then to find $u_{\varepsilon} \in K^{\perp}$ such that

$$
J_{\varepsilon}\left(u_{\varepsilon}\right)=\inf _{v \in K^{\perp}} J_{\varepsilon}(v) .
$$

As $K^{\perp}$ is a closed convex of $L^{2}\left(\omega_{T}\right)$, it is classical to show that there exists a unique solution $u_{\varepsilon}$ to $(75)$ (see for instance [3], Chapter 5, Section 3). If we write $y_{\varepsilon}=\left(y_{1 \varepsilon}, y_{2 \varepsilon}\right)$ the solution to (22), state associated to $u_{\varepsilon}$, using an adjoint state $\rho_{\varepsilon}=\left(\rho_{1 \varepsilon}, \rho_{2 \varepsilon}\right) \in\left(C\left([0, T] ; L^{2}(\Omega)\right) \cap L^{2}\left((0, T) ; H_{0}^{1}(\Omega)\right)\right)^{2}$, the triplet $\left(u_{\varepsilon}, y_{\varepsilon}, \rho_{\varepsilon}\right)$ is solution of the following first order optimality system:

$$
\begin{aligned}
& \left\{\begin{aligned}
\frac{\partial y_{1 \varepsilon}}{\partial t}-\Delta y_{1 \varepsilon}+a y_{1 \varepsilon}+b y_{2 \varepsilon} & =l+\left(u_{\varepsilon}-u_{0}\right) \chi_{\omega} & & \text { in } Q, \\
\frac{\partial y_{2 \varepsilon}}{\partial t}-\Delta y_{2 \varepsilon}+c y_{2 \varepsilon}+d y_{1 \varepsilon} & =0 & & \text { in } Q, \\
y_{1 \varepsilon}=y_{2 \varepsilon} & =0 & & \text { on } \Sigma, \\
y_{1 \varepsilon}(0)=y_{2 \varepsilon}(0) & =0 & & \text { in } \Omega,
\end{aligned}\right. \\
& \left\{\begin{aligned}
-\frac{\partial \rho_{1 \varepsilon}}{\partial t}-\Delta \rho_{1 \varepsilon}+a \rho_{1 \varepsilon}+d \rho_{2 \varepsilon} & =0 & & \text { in } \quad Q, \\
-\frac{\partial \rho_{2 \varepsilon}}{\partial t}-\Delta \rho_{2 \varepsilon}+c \rho_{2 \varepsilon}+b \rho_{1 \varepsilon} & =0 & & \text { in } \quad Q, \\
\rho_{1 \varepsilon}=\rho_{2 \varepsilon} & =0 & & \text { on } \quad \Sigma, \\
\rho_{1 \varepsilon}(T) & =\frac{1}{\varepsilon} y_{1 \varepsilon}(T) & & \text { in } \quad \Omega, \\
\rho_{2 \varepsilon}(T) & =\frac{1}{\varepsilon} y_{2 \varepsilon}(T) & & \text { on } \quad \Omega
\end{aligned}\right.
\end{aligned}
$$

and

$$
u_{\varepsilon}=\left(P \rho_{1 \varepsilon}-\rho_{1 \varepsilon}\right) \chi_{\omega_{T}} \in K^{\perp} .
$$

Multiplying the state equations $(76)_{1}$ and $(76)_{2}$ respectively by $\rho_{1 \varepsilon}$ and $\rho_{2 \varepsilon}$ solutions of (77), then integrating by parts over $Q$ and adding the resulting identities, we obtain

$$
\frac{1}{\varepsilon}\left\|y_{1 \varepsilon}(T)\right\|_{L^{2}(\Omega)}^{2}+\frac{1}{\varepsilon}\left\|y_{2 \varepsilon}(T)\right\|_{L^{2}(\Omega)}^{2}=\int_{Q} l \rho_{1 \varepsilon} d x d t+\int_{\omega_{T}}\left(u_{\varepsilon}-u_{0}\right) \rho_{1 \varepsilon} d x d t,
$$

so that

$$
\begin{array}{r}
\frac{1}{\varepsilon}\left\|y_{1 \varepsilon}(T)\right\|_{L^{2}(\Omega)}^{2}+\frac{1}{\varepsilon}\left\|y_{2 \varepsilon}(T)\right\|_{L^{2}(\Omega)}^{2}+\left\|u_{\varepsilon}\right\|_{L^{2}\left(\omega_{T}\right)}^{2}= \\
\int_{Q} l \rho_{1 \varepsilon} d x d t+\int_{\omega_{T}} u_{\varepsilon} P \rho_{1 \varepsilon} \chi_{\omega} d x d t-\int_{\omega_{T}} u_{0} \rho_{1 \varepsilon} d x d t .
\end{array}
$$


Since $u_{\varepsilon} \in K^{\perp}$, this gives

$$
2 J_{\varepsilon}\left(u_{\varepsilon}\right)=\int_{Q} l \rho_{1 \varepsilon} d x d t-\int_{\omega_{T}} u_{0} \rho_{1 \varepsilon} d x d t
$$

In view of $(78)$,

$$
u_{\varepsilon}=-(I-P) \rho_{1 \varepsilon} \chi_{\omega} .
$$

Thus,

$$
\begin{array}{r}
\frac{1}{\varepsilon}\left\|y_{1 \varepsilon}(T)\right\|_{L^{2}(\Omega)}^{2}+\frac{1}{\varepsilon}\left\|y_{2 \varepsilon}(T)\right\|_{L^{2}(\Omega)}^{2}+\left\|(I-P) \rho_{1 \varepsilon}\right\|_{L^{2}\left(\omega_{T}\right)}^{2}= \\
\int_{Q} l \rho_{1 \varepsilon} d x d t-\int_{\omega_{T}} u_{0} \rho_{1 \varepsilon} d x d t .
\end{array}
$$

This implies that

$$
\begin{aligned}
\frac{1}{\varepsilon}\left\|y_{1 \varepsilon}(T)\right\|_{L^{2}(\Omega)}^{2} & +\frac{1}{\varepsilon}\left\|y_{2 \varepsilon}(T)\right\|_{L^{2}(\Omega)}^{2}+\left\|(I-P) \rho_{1 \varepsilon}\right\|_{L^{2}\left(\omega_{T}\right)}^{2} \\
& \leq\left[\|l\|_{H_{\theta}}+\left\|\theta u_{0}\right\|_{L^{2}\left(\omega_{T}\right)}\right]\left\|\frac{1}{\theta} \rho_{1 \varepsilon}\right\|_{L^{2}(Q)} .
\end{aligned}
$$

Because $\rho_{\varepsilon}=\left(\rho_{1 \varepsilon}, \rho_{2 \varepsilon}\right)$ solution to $(77)$ belongs to $W$, we have from (38) that

$$
\left\|\frac{1}{\theta} \rho_{1 \varepsilon}\right\|_{L^{2}(Q)}^{2} \leq C\left\|\rho_{1 \varepsilon}-P \rho_{1 \varepsilon}\right\|_{L^{2}\left(\omega_{T}\right)}^{2} d x d t
$$

which combining with (79) gives

$$
\begin{array}{r}
\frac{1}{\varepsilon}\left\|y_{1 \varepsilon}(T)\right\|_{L^{2}(\Omega)}^{2}+\frac{1}{\varepsilon}\left\|y_{2 \varepsilon}(T)\right\|_{L^{2}(\Omega)}^{2}+\left\|(I-P) \rho_{1 \varepsilon}\right\|_{L^{2}\left(\omega_{T}\right)}^{2} \\
\leq C\left(\|l\|_{H_{\theta}}+\left\|\theta u_{0}\right\|_{L^{2}\left(\omega_{T}\right)}\right)\left\|(I-P) \rho_{1 \varepsilon}\right\|_{L^{2}\left(\omega_{T}\right)} .
\end{array}
$$

It then follows from (27) and (28) that

$$
\frac{1}{\varepsilon}\left\|y_{1 \varepsilon}(T)\right\|_{L^{2}(\Omega)}^{2}+\frac{1}{\varepsilon}\left\|y_{2 \varepsilon}(T)\right\|_{L^{2}(\Omega)}^{2}+\left\|(I-P) \rho_{1 \varepsilon}\right\|_{L^{2}\left(\omega_{T}\right)}^{2} \leq C\|l\|_{H_{\theta}}\left\|(I-P) \rho_{1 \varepsilon}\right\|_{L^{2}\left(\omega_{T}\right)} .
$$

Hence, we deduce that

$$
\begin{aligned}
\left\|(I-P) \rho_{1 \varepsilon}\right\|_{L^{2}\left(\omega_{T}\right)} & \leq C\|l\|_{H_{\theta}}, \\
\left\|y_{1 \varepsilon}(T)\right\|_{L(\Omega)} & \leq C \sqrt{\varepsilon}\|l\|_{H_{\theta}}, \\
\left\|y_{2 \varepsilon}(T)\right\|_{L(\Omega)} & \leq C \sqrt{\varepsilon}\|l\|_{H_{\theta}}, \\
\left\|u_{\varepsilon}\right\|_{L^{2}\left(\omega_{T}\right)}^{2} & \leq C\|l\|_{H_{\theta}},
\end{aligned}
$$

where $C>0$ is independent of $\varepsilon$. Then from the properties of the system (76) we get

$$
\left\|y_{\varepsilon}\right\|_{\left(L^{2}\left((0, T) ; H_{0}^{1}(\Omega)\right)\right)^{2}} \leq C\|l\|_{H_{\theta}} .
$$


From (80), (81a), we have on the one hand,

$$
\left\|\frac{1}{\theta} \rho_{1 \varepsilon}\right\|_{L^{2}(Q)} \leq C\|l\|_{H_{\theta}},
$$

and on the other hand, because $\frac{1}{\theta} \in L^{\infty}(Q)$,

$$
\left\|\frac{1}{\theta} P \rho_{1 \varepsilon}\right\|_{L^{2}\left(\omega_{T}\right)} \leq C\|l\|_{H_{\theta}} .
$$

Therefore, $K$ being a finite dimensional vector subspace of $L^{2}\left(\omega_{T}\right)$, we deduce that

$$
\left\|P \rho_{1 \varepsilon}\right\|_{L^{2}\left(\omega_{T}\right)} \leq C\|l\|_{H_{\theta}}
$$

Using again (81a) we obtain

$$
\left\|\rho_{1 \varepsilon}\right\|_{L^{2}\left(\omega_{T}\right)} \leq C\|l\|_{H_{\theta}} .
$$

From the definition of the norm on $W$ given by (53), we can write

$$
\left\|\rho_{\varepsilon}\right\|_{W}^{2}=\left\|\rho_{1 \varepsilon}-P \rho_{1 \varepsilon}\right\|_{L^{2}\left(\omega_{T}\right)}^{2},
$$

because $(77)_{1}$ and $(77)_{2}$ hold. Hence, using (81a), we deduce that

$$
\left\|\rho_{\varepsilon}\right\|_{W} \leq C\|l\|_{H_{\theta}} .
$$

Step 3. We pass to the limit in the first order optimality system (76)-(78). In view of (81) and (82), we can extract subsequences of $\left(v_{\varepsilon}\right)$ and $\left(y_{\varepsilon}\right)=$ $\left(\left(y_{1 \varepsilon}\right),\left(y_{2 \varepsilon}\right)\right)$ (still denoted $\left(v_{\varepsilon}\right)$ and $\left(y_{\varepsilon}\right)=\left(\left(y_{1 \varepsilon}\right),\left(y_{2 \varepsilon}\right)\right)$ such that

$$
\begin{aligned}
u_{\epsilon} & \rightarrow v_{0} \text { weakly in } L^{2}\left(\omega_{T}\right), \\
\left(y_{1 \varepsilon}(T), y_{2 \varepsilon}(T)\right) & \rightarrow(0,0) \text { strongly in } L^{2}(\Omega) \times L^{2}(\Omega), \\
y_{1 \varepsilon} & \rightarrow y_{10} \text { weakly in } L^{2}\left((0, T), H_{0}^{1}(\Omega)\right), \\
y_{2 \varepsilon} & \rightarrow y_{20} \text { weakly in } L^{2}\left((0, T), H_{0}^{1}(\Omega)\right) .
\end{aligned}
$$

Proceeding as for $y_{\theta}=\left(y_{1 \theta}, y_{2 \theta}\right)$ at the pages 17 and 18 , we prove that $\left(v_{0},\left(y_{10}, y_{20}\right)\right)$ is solution of (21)-( 23).

Actually, $\left(v_{0}, y_{0}\right)=(\hat{v}, \hat{y})$ where $\hat{v}$ is the optimal control solution of (72) and $\hat{y}=\hat{y}(\hat{v})$ is the solution to (22) corresponding to $\hat{v}$. Indeed, from the expression of $J_{\varepsilon}$ given by (74), we can write

$$
\frac{1}{2}\left\|u_{\varepsilon}\right\|_{L^{2}\left(w_{T}\right)}^{2} \leq J_{\varepsilon}\left(u_{\varepsilon}\right) .
$$

Because $(\hat{v}, \hat{y})$ is solution of $(21)-(23)$, it follows from $(75)$ that

$$
J_{\varepsilon}\left(u_{\varepsilon}\right) \leq \frac{1}{2}\|\hat{v}\|_{L^{2}\left(w_{T}\right)}^{2} .
$$


We then deduce from these two latter inequalities that

$$
\frac{1}{2}\left\|u_{\varepsilon}\right\|_{L^{2}\left(w_{T}\right)}^{2} \leq J_{\varepsilon}\left(u_{\varepsilon}\right) \leq \frac{1}{2}\|\hat{v}\|_{L^{2}\left(w_{T}\right)}^{2} .
$$

Then, using (87) while passing in the limit in (91), we obtain that

$$
\frac{1}{2}\left\|v_{0}\right\|_{L^{2}\left(w_{T}\right)}^{2} \leq \liminf _{n \rightarrow \infty} J_{\varepsilon}\left(u_{\varepsilon}\right) \leq \frac{1}{2}\|\hat{v}\|_{L^{2}\left(w_{T}\right)}^{2} .
$$

Consequently,

$$
\left\|v_{0}\right\|_{L^{2}\left(\omega_{T}\right)}^{2} \leq\|\hat{v}\|_{L^{2}\left(\omega_{T}\right)}^{2} .
$$

As $\hat{v}$ is solution of (72), we deduce that $\hat{v}=v_{0}$. And, since (22) admits a unique solution, we have $y_{0}=\hat{y}$. This means that $(\hat{v}, \hat{y})$ satisfies

$$
\left\{\begin{aligned}
\frac{\partial \hat{y}_{1}}{\partial t}-\Delta \hat{y}_{1}+a \hat{y}_{1}+b \hat{y}_{2} & =l+\left(\hat{v}-u_{0}\right) \chi_{\omega} & & \text { in } Q, \\
\frac{\partial y_{2}}{\partial t}-\Delta \hat{y}_{2}+c \hat{y}_{2}+d \hat{y}_{1} & =0 & & \text { in } Q, \\
\hat{y}_{1}=\hat{y}_{2} & =0 & & \text { on } \Sigma, \\
\hat{y}_{1}(0)=\hat{y}_{2}(0) & =0 & & \text { in } \Omega .
\end{aligned}\right.
$$

Then from (88), we have that

$$
\hat{y}_{1}(T)=\hat{y}_{2}(T)=0 \text { in } \Omega .
$$

Now, in view of (83) and (86), we can extract subsequences of $\left(\rho_{1 \varepsilon}\right)$ and $\left(\rho_{2 \varepsilon}\right)$ (still denoted $\left(\rho_{1 \varepsilon}\right)$ and $\left(\rho_{2 \varepsilon}\right)$ ) such that

$$
\begin{aligned}
\rho_{1 \varepsilon} & \rightarrow \rho_{1} \text { weakly in } D^{\prime}(Q), \\
\rho_{2 \varepsilon} & \rightarrow \rho_{1} \text { weakly in } D^{\prime}(Q), \\
\rho_{\varepsilon} & \rightarrow \rho \text { weakly in } W .
\end{aligned}
$$

From (94a) and (94b), we have that

$$
\begin{array}{rlr}
-\frac{\partial \rho_{1 \varepsilon}}{\partial t}-\Delta \rho_{1 \varepsilon}+a \rho_{1}+d \rho_{2 \varepsilon} & \rightarrow-\frac{\partial \rho_{1}}{\partial t}-\Delta \rho_{1}+a \rho_{1}+d \rho_{2} & \text { weakly in } D^{\prime}(Q), \\
-\frac{\partial \rho_{2 \varepsilon}}{\partial t}-\Delta \rho_{2 \varepsilon}+c \rho_{2 \varepsilon}+b \rho_{1 \varepsilon} & \rightarrow-\frac{\partial \rho_{2}}{\partial t}-\Delta \rho_{2}+c \rho_{2}+b \rho_{1} & \text { weakly in } D^{\prime}(Q) .
\end{array}
$$

It then follows from $(77)_{1},(77)_{2}$ and (94c) that $\rho=\left(\rho_{1}, \rho_{2}\right) \in W$ satisfies

$$
\left\{\begin{array}{rll}
-\frac{\partial \rho_{1}}{\partial t}-\Delta \rho_{1}+a \rho_{1}+d \rho_{2} & =0 & \text { in } Q \\
-\frac{\partial \rho_{2}}{\partial t}-\Delta \rho_{2}+c \rho_{2}+b \rho_{1} & =0 & \text { in } Q \\
\rho_{1}=\rho_{2} & =0 & \text { on } \Sigma .
\end{array}\right.
$$

In view of (85), we can write

$$
\rho_{1 \varepsilon} \rightarrow \rho_{1} \text { weakly in } L^{2}\left(\omega_{T}\right) .
$$


As $P$ is a continuous and compact operator, we deduce that

$$
P \rho_{1 \varepsilon} \rightarrow P \rho_{1} \text { weakly in } L^{2}\left(\omega_{T}\right) .
$$

Consequently,

$$
u_{\varepsilon}=(P-I) \rho_{1 \varepsilon} \chi_{\omega} \rightarrow(P-I) \rho_{1} \chi_{\omega} \text { weakly in } L^{2}\left(\omega_{T}\right) .
$$

Thus

$$
\hat{v}=-(I-P) \rho_{1} \chi_{\omega} \in K^{\perp} .
$$

\subsection{Proof of Theorem $\mathbf{1 . 3}$}

We showed that there exists a unique control $\hat{v}=(P-I) \rho_{1} \chi_{\omega} \in K^{\perp}$ such that $(\hat{v}, \hat{y})$ verifies the null controllability problem with constraint on the control (21)-(23). Consequently, Proposition 2.1 let us say that the control $k=-u_{0}+$ $(P-I) \rho_{1} \chi_{\omega}$ is solution of the null controllability problem with state constraints (1), (7), (8). Furthermore, as $k=\hat{v}-u_{0}$, using estimates (28) and (73), we obtain

$$
\|k\|_{L^{2}\left(\omega_{T}\right)} \leq C\|l\|_{H_{\theta}} .
$$

\section{Study of Problem 2}

We need some preliminary results, useful for the proof of Theorem 1.4.

\subsection{Preliminary results}

Lemma 3.1 Let $u_{0}$ be defined by relation (25). Then $u_{0} \in H_{\theta}$ and the map $F_{1}$ defined by

$$
\begin{aligned}
F_{1}: H_{\theta} & \rightarrow L^{2}\left(\omega_{T}\right) \\
l & \mapsto F_{1}(l)=u_{0}(l) \chi_{\omega}
\end{aligned}
$$

is linear and continuous.

Proof. Because $u_{0} \in K_{\theta} \subset L^{2}\left(\omega_{T}\right)$, the application $F_{1}$ is well defined. From relation (25) we have that the application $F_{1}$ is linear, and continuous since (28) holds.

Lemma 3.2 Let $H_{\theta}$ be defined by (4). Then for any $l \in H_{\theta}$, the map

$$
\begin{aligned}
F_{2}: H_{\theta} & \rightarrow L^{2}\left(\omega_{T}\right) \\
l & \mapsto F_{2}(l)=-\left(\rho_{1}-P \rho_{1}\right) \chi_{\omega}
\end{aligned}
$$

where $\rho=\left(\rho_{1}, \rho_{2}\right)=\left(\rho_{1}(l), \rho_{2}(l)\right)$ is solution of (95), is linear and continuous. 
Proof. Let $W$ be the Hilbert space defined by (52). Set

$$
W_{0}=\left\{\varphi=\left(\varphi_{1}, \varphi_{2}\right) \in W=V \times V \text { such that } \mathcal{M}(\varphi)=\mathcal{N}(\varphi)=0\right\} .
$$

Then $W_{0}$ is a Hilbert space since $W_{0}$ is a closed subspace of $W$.

Multiplying the state equations $(92)_{1}$ and $(92)_{2}$ respectively by $\varphi_{1}$ and $\varphi_{2}$, integrating them by parts over $Q$ and adding the resulting identities, we obtain

$$
\begin{aligned}
\int_{Q} \varphi_{1}\left(l+\left(\hat{v}(l)-u_{0}(l)\right) \chi_{\omega}\right) d x d t & =\int_{\Omega} \hat{y}_{1}(T) \varphi_{1}(T) d x \\
& +\int_{\Omega} \hat{y}_{2}(T) \varphi_{2}(T) d x, \quad \forall\left(\varphi_{1}, \varphi_{2}\right) \in W_{0},
\end{aligned}
$$

which in view of (93) gives

$$
\int_{\omega_{T}} \varphi_{1} \hat{v}(l) d x d t=\int_{Q} \varphi_{1}\left(u_{0}(l) \chi_{\omega}-l\right) d x d t, \quad \forall\left(\varphi_{1}, \varphi_{2}\right) \in W_{0} .
$$

On the other hand, using the fact that $\hat{v}(l) \in K^{\perp}$ and the definition of the bilinear form $\mathcal{B}$ given by (51), we have

$$
\begin{aligned}
\int_{\omega_{T}} \varphi_{1} \hat{v}(l) d x d t & =\int_{\omega_{T}}\left(P \rho_{1}(l)-\rho_{1}(l)\right) \varphi_{1} d x d t \\
& =\int_{\omega_{T}}\left(\rho_{1}-P \rho_{1}\right)\left(\varphi_{1}-P \varphi_{1}\right) d x d t \\
& =\mathcal{B}(\rho, \varphi), \quad \forall \varphi=\left(\varphi_{1}, \varphi_{2}\right) \in W_{0} .
\end{aligned}
$$

As the linear form $\mathcal{L}$ defined by (54) is also continuous on $W_{0}$, we deduce that there exists a unique $\rho=\left(\rho_{1}, \rho_{2}\right) \in W_{0}$ solution of the variational problem:

$$
\mathcal{B}(\rho, \varphi)=\int_{Q}\left(l-u_{0} \chi_{\omega}\right) \varphi_{1} d x d t, \quad \forall \varphi=\left(\varphi_{1}, \varphi_{2}\right) \in W_{0} .
$$

Consequently, the map $l \mapsto \rho(l)$ is linear from $H_{\theta}$ into $L^{2}\left(\omega_{T}\right)$ and because the operator $I-P$ defined from $L^{2}\left(\omega_{T}\right)$ into $K^{\perp} \subset L^{2}\left(\omega_{T}\right)$ is linear, we deduce that $F_{2}$ is linear. The continuity of $F_{2}$ is straightforward from estimate (73).

Remark 2 It follows from the definitions of the maps $F_{1}$ and $F_{2}$ given respectively by (98) and (99) that the map

$$
\begin{aligned}
F: H_{\theta} & \rightarrow L^{2}\left(\omega_{T}\right) \\
l & \mapsto F(l)=\left(\hat{v}(l)-u_{0}(l)\right) \chi_{\omega},
\end{aligned}
$$

is linear and continuous. 


\subsection{Proof of Theorem 1.4}

Let $H_{\theta}$ be defined by $(4)$ and $\left(\hat{v}, \hat{y}=\left(\hat{y}_{1}, \hat{y}_{2}\right)\right)$ be the solution of $(92),(93)$ and (96)( or equivalently (1), (7) and (8)).

Consider the cost function $J$ defined by

$$
J(l)=\frac{1}{2}\left\|y_{1}(l)-z_{d}^{1}\right\|_{L^{2}(Q)}^{2}+\frac{1}{2}\left\|y_{2}(l)-z_{d}^{2}\right\|_{L^{2}(Q)}^{2}+\frac{N}{2}\|l\|_{H_{\theta}}^{2}
$$

from which we associate the minimization problem

$$
\inf _{l \in \mathcal{U}_{a d}} J(l),
$$

where $\mathcal{U}_{a d}$ is a non empty closed convex subspace of $H_{\theta}$.

From the properties of the map $F$ defined in Remark 2, we have that $J$ is strictly convex, continuous and coercive. Consequently, the optimal control problem (102) has a unique control $\hat{l} \in \mathcal{U}_{a d}$. To characterize this optimal control, we write the Euler-Lagrange conditions:

$$
\frac{d}{d \lambda} J(\hat{l}+\lambda(l-\hat{l}))_{\mid \lambda=0} \geq 0, \forall l \in \mathcal{U}_{a d}
$$

After some calculations, we obtain

$$
\begin{array}{r}
\left(\hat{y}_{1}-z_{d}^{1}, y_{1}(l-\hat{l})\right)_{L^{2}(Q)}+\left(\hat{y}_{2}-z_{d}^{2}, y_{2}(l-\hat{l})\right)_{L^{2}(Q)} \\
+N(\hat{l}, l-\hat{l})_{H_{\theta}} \geq 0, \forall l \in \mathcal{U}_{a d}
\end{array}
$$

where

$w=l-\hat{l}$ and the corresponding state $\left(q_{1}, q_{2}\right)=\left(y_{1}(l-\hat{l}), y_{2}(l-\hat{l})\right)$ satisfy

$$
\left\{\begin{aligned}
\frac{\partial q_{1}}{\partial t}-\Delta q_{1}+a q_{1}+b q_{2} & =w+\left(\hat{v}(w)-u_{0}(w)\right) \chi_{\omega} & & \text { in } \quad Q \\
\frac{\partial q_{2}}{\partial t}-\Delta q_{2}+c q_{2}+d q_{1} & =0 & & \text { in } \quad Q \\
q_{1}=q_{2} & =0 & & \text { on } \Sigma \\
q_{1}(0)=q_{2}(0) & =0 & & \text { in } \Omega .
\end{aligned}\right.
$$

To interpret (103), we use the adjoint system

$$
\left\{\begin{array}{rlll}
-\frac{\partial p_{1}}{\partial t}-\Delta p_{1}+a p_{1}+d p_{2} & =\hat{y}_{1}-z_{d}^{1} & & \text { in } \quad Q \\
-\frac{\partial p_{2}}{\partial t}-\Delta p_{2}+c p_{2}+b p_{1} & =\hat{y}_{2}-z_{d}^{2} & & \text { in } \quad Q \\
p_{1}=p_{2} & =0 & & \text { on } \quad \Sigma \\
p_{1}(T)=p_{2}(T) & =0 & \text { in } \quad \Omega .
\end{array}\right.
$$


Because $\left(\hat{y}_{1}-z_{d}^{1}, \hat{y}_{2}-z_{d}^{2}\right) \in\left[L^{2}(Q)\right]^{2}$, system (105) has a unique solution $p=\left(p_{1}, p_{2}\right) \in\left[C\left([0, T] ; L^{2}(\Omega)\right) \cap L^{2}\left((0, T) ; H_{0}^{1}(\Omega)\right)\right]^{2}$.

Multiplying ( 105) $)_{1}$ and $(105)_{2}$ respectively by $q_{1}$ and $q_{2}$, integrating them by parts over $Q$ and adding the resulting identities, we obtain

$$
\begin{aligned}
\int_{Q} q_{1}\left(\hat{y}_{1}-z_{d}^{1}\right) d x d t+\int_{Q} q_{2}\left(\hat{y}_{2}-z_{d}^{2}\right) d x d t= & \int_{Q} p_{1}\left(\frac{\partial q_{1}}{\partial t}-\Delta q_{1}+a q_{1}+b q_{2}\right) d x d t \\
& +\int_{Q} p_{2}\left(\frac{\partial q_{2}}{\partial t}-\Delta q_{2}+c q_{2}+d q_{1}\right) d x d t
\end{aligned}
$$

which in view of (104) gives

$$
\begin{aligned}
\int_{Q} q_{1}\left(\widehat{y_{1}}-z_{d}^{1}\right) d x d t+\int_{Q} q_{2}\left(\widehat{y_{2}}-z_{d}^{2}\right) d x d t & =\int_{Q} p_{1}\left(w+\left(\hat{v}(w)-u_{0}(w)\right) \chi_{\omega}\right) d x d t \\
& =\int_{Q} p_{1} w d x d t+\int_{\omega_{T}} p_{1} F(w) d x d t
\end{aligned}
$$

where $F$ is the application defined in Remark 2.

Let $H_{\theta}{ }^{\prime}$ be the dual of Hilbert space $H_{\theta}$. Let also $\Lambda^{-1}$ be the isometric isomorphism from $H_{\theta}{ }^{\prime}$ to $H_{\theta}$.

We have

$$
\begin{aligned}
\int_{Q} p_{1} w d x d t & =\int_{Q}\left(\frac{1}{\theta} p_{1}\right)(\theta w) d x d t \\
& =\left\langle\frac{1}{\theta} p_{1}, w\right\rangle_{H_{\theta^{\prime}}, H_{\theta}} \\
& =\left(\Lambda^{-1}\left(\frac{1}{\theta} p_{1}\right), w\right)_{H_{\theta}}
\end{aligned}
$$

and

$$
\begin{aligned}
\int_{\omega_{T}} p_{1} F(w) d x d t & =\int_{Q} F^{*}\left(p_{1} \chi_{\omega_{T}}\right) w d x d t \\
& =\left\langle\frac{1}{\theta} F^{*}\left(p_{1} \chi_{\omega_{T}}\right), w\right\rangle_{H_{\theta^{\prime}, H_{\theta}}} \\
& =\left(\Lambda^{-1}\left(\frac{1}{\theta} F^{*}\left(p_{1} \chi_{\omega_{T}}\right)\right), w\right)_{H_{\theta}} .
\end{aligned}
$$

Hence

$$
\int_{Q} q_{1}\left(\widehat{y_{1}}-z_{d}^{1}\right) d x d t+\int_{Q} q_{2}\left(\widehat{y_{2}}-z_{d}^{2}\right) d x d t=\left(\Lambda^{-1}\left(\frac{1}{\theta} p_{1}\right)+\Lambda^{-1}\left(\frac{1}{\theta} F^{*}\left(p_{1} \chi_{\omega_{T}}\right)\right), w\right)_{H_{\theta}} .
$$

Finally, (103) and (106) give

$$
\left(\Lambda^{-1}\left(\frac{1}{\theta} p_{1}+\frac{1}{\theta} F^{*}\left(p_{1} \chi_{\omega_{T}}\right)\right)+N \hat{l}, l-\hat{l}\right)_{H_{\theta}} \geq 0, \quad \forall l \in \mathcal{U}_{a d}
$$


Acknowledgments: The authors would like to express their gratitude to the unknown referee for helpful advice. His many helpful comments have led to improvement the manuscript.

\section{References}

[1] A. Fursikov \& O. Imanuvilov. Controllability of evolution equations: Lecture Notes, vol.34, Research Institute of Mathematics, Seoul National University, Korea (1996).

[2] A. V. Fursikov. Optimal Control of Distributed Systems. Theory and Applications, volume 187. American Mathematical Society, Providence, RI, 2000.

[3] J. L. Lions. Contrôle optimal de systèmes gouvernés par des équations aux dérivées partielles, Dunod, Paris (1968).

[4] J. L. Lions. Sentinelles pour les systèmes distribués à données incomplétes. Masson, Paris,(1992).

[5] J. L. Lions. Contrôlabilité exacte, perturbation et stabilisation de systèmes distribués 1, Rech. Math. Appl. 8, Masson, Paris, (1988).

[6] J. L. Lions and M. Magenes, Problèmes aux limites non homogènes et applications. Dunod, vol.1 et 2, Paris 168. Zb1 0165.10801 MR 1159093.

[7] O. Nakoulima, Contrôlabilité à zéro avec contraintes sur le contrôle, C.R. Acad. Sci. Paris Ser. I 339 (2004) 405-410.

[8] O. Nakoulima, Optimal control for distributed systems subject to nullcontrollability. Application to discriminating sentinels, ESAIM: COCV 13 (4) (2007) 623-638.

[9] M. Mercan. Optimal control for distributed linear systems subjected to nullcontrollability. Applicable Analysis, Vol. 92, issue 9, pp 1928-1943.

[10] M. Mercan. Optimal Control for Distributed Linear Systems Subjected to Null Controllability with Constraints on the State. Vol. 37 of the series Springer Proceedings in Mathematics \& Statistics pp 213-232.

[11] G.M. Mophou, Null controllability with constraints on the state for nonlinear heat equations. Forum Mathematicum. (2011), 23(2): 285-319.

[12] G.M. Mophou, O. Nakoulima, Null controllability with constraints on the state for the semilinear heat equation. Journal of optimization theory and applications, 2009, 143(3): 539-565.

[13] M. Mercan \& O. Nakoulima. Control of Stackelberg for a two stroke problem. Dynamics of continuous, Discrete and Impulsive systems: Applications \& Algorithms 22 (2015), 441-463 
[14] M. Mercan \& G. M. Mophou. Null controllability with state constraints of a linear backward population dynamics problem. Internal journal of evolution equations, 9(1), p 99-120, (2014).

[15] C. Louis-Rose. Simultaneous null controllability with constraint on the control. Applied Mathematics and Computation, 219 (2013), 6372-6392.

[16] C. Louis-Rose, A null controllability problem with a nite number of constraints on the normal derivative for the semilinear heat equation. E. J. Qualitative Theory of Di . Equ, 2012, 95: 1-34.

[17] D.L. Russell, The Dirichlet-Neumann boundary control problem associated with Maxwell's equations in a cylindrical region, SIAM J. Control Optim. 24 (1986) 199-229.

[18] G.O. Antunes, F.A. Araruna, and L.A. Medeiros. Simultaneous controllability for a system with resistance term. Tendências em Matemàtica Aplicada e Computacional, 3(1) :31- 40, 2002.

[19] B.V. Kapitonov and G.P. Menzala. Simultaneous exact controllability for Maxwell equations and for a second-order hyperbolic system. Electronic Journal of Differential

Equations, 2010(24) :1-13, 2010.

[20] L.C. Evans, Partial Differential Equations, American Mathematical Society, Providence, Rhode Island, 1998.

[21] F. Ammar Khodja, A. Benabdallah, C. Dupaix, Null-controllability of some reaction-diffusion systems with one control force, J. Math. Anal. Appl. 320 (2006) 928-943.

[22] P. Gao. Null controllability with constraints on the state for the reaction-diffusion system. Computers \& Mathematics with applications. doi:10.1016/j.camwa.2015.05.027. 